\title{
Measurement Errors in Dynamic Models
}

\author{
Ivana Komunjer* Serena $\mathrm{Ng}^{\dagger}$
}

April 15, 2011

\begin{abstract}
Static models that are not identifiable in the presence of white noise measurement errors are known to be potentially identifiable when the model has dynamics. However, few results are available for the plausible case of serially correlated measurement errors. This paper provides order and rank conditions for "limited information" identification of parameters in dynamic models with measurement errors where some aspects of the probability model are not fully specified or utilized. The key is to consider a model for the contaminated data that has richer dynamics than the model for the correctly observed data. Simply counting the total number of unknown parameters in the true model relative to the estimable model will not yield an informative order condition for identification. Implications for single equation, VARs, and panel data models are studied.
\end{abstract}

Keywords: dynamic models, errors-in-variables, identification

JEL Classification: C32, C19

\footnotetext{
*University of California, San Diego, 9500 Gilman Drive, La Jolla, CA 92093. Email: komunjer@ucsd.edu

${ }^{\dagger}$ Columbia University, 420 W. 118 St. MC 3308, New York, NY 10027. Email: serena.ng@columbia.edu This paper was presented at the 2010 SETA conference in Singapore. We thank the editor, three anonymous referees and the seminar participants for helpful comments. Financial support from the National Science Foundation SES0962473 and SES-0962431 is gratefully acknowledged.
} 


\section{Introduction}

Empirical analysis often involves using an observable that is an inexact measure of the latent variable of interest. To distinguish the marginal effect of a covariate from measurement error, assumptions on the measurement error process are required. While the white noise assumption is convenient, it is rather restrictive in time series and panel data settings when the data are correlated over time. This paper provides general conditions for identification of dynamic models subject to serially correlated measurement errors. It is shown that the error-ridden data necessitate consideration of a model with higher order dynamics than if the data were correctly observed. Furthermore, an informative order condition for identification cannot be obtained by simply comparing the total number of unknowns in the true model with that of the estimable model. Specific conditions are provided for vector autoregressive models with predetermined variables (VARX), and dynamic panel models with fixed effects.

As is well documented, measurement errors are prevalent in survey data. Erickson and Whited (2000) argue that investment cash-flow regressions can be uninformative when Tobin's $Q$ is measured with errors because average $Q$ is no longer a good proxy for marginal $Q$. Devereux (2001) finds significant errors in survey reports of earnings and work hours. Quality variables (such as that of hospitals and the environment) are especially susceptible to measurement errors. Aggregate data are less susceptible but are not immune to measurement error problems. Since we do not observe variables such as the level of global economic activity or the state of economy, filtered variables are often used as proxies. Except by coincidence, the deviations between the latent process and the filtered series are likely to be serially correlated. Furthermore, errors in data collection and reporting are inevitable. Wilcox (1992) discusses the issues in consumption measurements especially at the monthly level. If the theoretical construct of consumption differs from its empirical counterpart, identification of the behavioral parameters and testing of consumption from observed data will be misleading. Ermini (1993) shows that allowing for serially uncorrelated measurement errors changes the measure of persistence in consumption growth. Falk and Lee (1990) suggest that measurement errors can explain rejections of the permanent income hypothesis. Sargent (1989) considers a model in which the data collecting agency observes data with serially correlated errors but reports a filtered version of the data. In the context of an accelerator model, he shows that when the data are error-ridden, more steps are needed to estimate the parameters.

Given that economic time series are known to be persistent, it is somewhat surprising that measurement errors are usually assumed to be serially uncorrelated. Perhaps one explanation is that estimation with white noise measurement errors is no more difficult than when measurement errors are absent provided valid instruments can be found. However, when measurement errors are 
serially correlated, short lags of the data will still be correlated with the composite error while lags long enough to satisfy instrument exogeneity will likely be weak. Eberly, Rebelo, and Vincent (2009) estimate an $\mathrm{AR}(1)$ model for the measurement noise in Tobin's $Q$ and note that measurement error problem "cannot be corrected in investment regressions using instrumental regressions." Dealing with correlated measurement errors might well require state space modeling of all variables which may not always be possible.

Indeed, while much has been written about errors-in-variables (EIV) in a static setting, the role of measurement errors in dynamic models is much less studied. Grether and Maddala (1973) examine the effects of measurement errors on the probability limits of the estimated coefficients in distributed lag models. They show that measurement errors in the exogenous variables may lead a researcher to detect long lags in adjustments. Ashley and Vaughan (1986) obtain an upper bound on the amount of measurement error in time series using a spectral decomposition method. Chanda (1996) studies the asymptotic properties of estimators based on Yule-Walker equations in autoregressive models with errors-in-variables. Tanaka (2002) develops tests to detect the existence of measurement errors in long and short memory data. Staudenmayer and Buonaccorsi (2005) study the estimation of parameters in autoregressive models when the measurement errors are uncorrelated but possibly heteroskedastic. All of the above papers focus on single equation analysis. Patriota, Sato, and Blas (2009) show that not accounting for measurement errors distorts Granger causality tests and consider estimation of VARs with white noise measurement errors.

We consider identification of single and multiple equation dynamic models with dynamic measurement errors under limited information. This can mean using the conditional model alone for identification leaving the marginal distribution of the exogenous variables unspecified, as in autoregressive distributed lag models. Or it can mean that some but not all aspects of the probability distribution of the data are used for identification. To proceed, Section 2 sets up the VARX framework and reviews related work in the literature. Section 3 first presents general order conditions for identification and then specializes to ARX and VAR models. Rank conditions are derived in Section 4 , and the results are illustrated via an example. Dynamic panel data models with measurement errors are considered in Section 5. Proofs are relegated to an Appendix. As a matter of notation, if $P(L)$ is an autoregressive polynomial matrix of order $p$, then $P(L)=I-\sum_{j=1}^{p} P_{j} L^{j}$ and if $Q(L)$ is a moving average polynomial matrix of order $q$, then $Q(L)=I+\sum_{j=1}^{q} Q_{j} L^{j}$. Finally, if $R(L)$ is a polynomial matrix of order $r$ associated with the covariates, then $R(L)=\sum_{j=0}^{r} R_{j} L^{j}$. 


\section{VARX Models with Measurement Errors}

Consider a system of dynamic equations relating $n_{y}$ endogenous variables $y_{t}=\left(y_{1 t}, \ldots, y_{n_{y}}\right)^{\prime}$ to $n_{x}$ exogenous variables $x_{t}=\left(x_{1 t}, \ldots, x_{n_{x}}\right)^{\prime}$ and $n_{y}$ disturbances $u_{t}=\left(u_{1 t}, \ldots, u_{n_{y}}\right)^{\prime}$. For all $t$, the data generating process (DGP) is assumed to be:

$$
y_{t}=\sum_{j=1}^{p_{y}} A_{j} y_{t-j}+\sum_{j=0}^{r_{x}} B_{j} x_{t-j}+u_{t} .
$$

We will henceforth refer to the DGP as Model-y. Then $\vec{A} \equiv\left(A_{1}, \ldots, A_{p_{y}}\right), \vec{B} \equiv\left(B_{0}, \ldots, B_{r_{x}}\right)$ and $\Sigma_{u}$ are the parameters of Model-y.

Suppose the observed data are contaminated by errors $\epsilon_{t}^{y}$ and $\epsilon_{t}^{x}$,

$$
\begin{aligned}
Y_{t} & =y_{t}+\epsilon_{t}^{y} \\
X_{t} & =x_{t}+\epsilon_{t}^{x} .
\end{aligned}
$$

A correctly measured variable has a corresponding measurement error component of zero. Let $M^{y}$ and $M^{x}$ be matrices consisting of zeros and ones (assumed known to the econometrician) that select the $n_{\epsilon}^{y}$ and $n_{\epsilon}^{x}$ nonzero components of $\epsilon_{t}^{y}$ and $\epsilon_{t}^{x}$, respectively. When the $j$-th component of $y_{t}$ is measured without error, the corresponding component of $\epsilon_{t}^{y}$ is zero, as is the $j$-th column of the $n_{\epsilon}^{y} \times n_{y}$ matrix $M^{y}$. When every component of $y_{t}$ is measured with error, $M^{y}$ is an identity matrix of dimension $n_{y}$.

Assumption A: (i) $u_{t} \sim W N\left(0, \Sigma_{u}\right)$ with $\Sigma_{u}$ full rank; (ii) $\left\{x_{t}\right\}$ is weakly stationary; (iii) for all $t$ and $\tau \geqslant 0, E\left[u_{t} x_{t-\tau}^{\prime}\right]=0$; (iv) for all $(t, s), E\left[\epsilon_{t}^{y} u_{s}^{\prime}\right]=0, E\left[\epsilon_{t}^{y} x_{s}^{\prime}\right]=0, E\left[\epsilon_{t}^{x} x_{s}^{\prime}\right]=0$, and $E\left[\epsilon_{t}^{x} u_{s}^{\prime}\right]=0$.

Assumption B: The matrix polynomial $A(L)$ is stable; $p_{y}$ and $r_{x}$ are known.

Assumption C: Let $v_{t}^{y} \sim W N\left(0, \Sigma_{v}^{y}\right)$ and $v_{t}^{x} \sim W N\left(0, \Sigma_{v}^{x}\right)$, where $\Sigma_{v}^{y}$ and $\Sigma_{v}^{x}$ are full rank diagonal matrices with $E\left[v_{t}^{y} v_{s}^{x \prime}\right]=0$ for all $(t, s)$. The measurement errors follow:

$$
\begin{aligned}
\Phi^{y}(L) M^{y} \epsilon_{t}^{y} & =\Theta^{y}(L) v_{t}^{y}, \\
\Phi^{x}(L) M^{x} \epsilon_{t}^{x} & =\Theta^{x}(L) v_{t}^{x},
\end{aligned}
$$

where $\Phi^{y}(L), \Theta^{y}(L), \Phi^{x}(L)$, and $\Theta^{x}(L)$ are diagonal polynomial matrices of known orders $p_{\epsilon}^{y}, q_{\epsilon}^{y}$, $p_{\epsilon}^{x}$, and $q_{\epsilon}^{x}$. Furthermore, $\Phi^{y}(L)$ and $\Phi^{x}(L)$ are stable; $\Phi^{y}(L)$ and $\Theta^{y}(L)$ as well as $\Phi^{x}(L)$ and $\Theta^{x}(L)$ are left co-prime. 
Under Assumption A, the vector white noise process $\left\{u_{t}\right\}$ is orthogonal to the measurement errors $\left\{\epsilon_{t}^{y}\right\}$ and $\left\{\epsilon_{t}^{x}\right\}$, and the latter are 'classical' in the sense that $\epsilon_{t}^{y}$ is uncorrelated with $y_{t}$, and $\epsilon_{t}^{x}$ is uncorrelated with $x_{t}$. Moreover, $u_{t}$ is uncorrelated with the current and past values of $x_{t}$ which is the usual exogeneity condition. Under Assumption $\mathrm{B}$, all roots of $\operatorname{det} A(z)(z \in$ $\mathbb{C}$ ) are outside the closed unit disk. Under Assumption $\mathrm{C}$, each element in $M^{y} \epsilon_{t}^{y}$ is a scalar $\operatorname{ARMA}\left(p_{\epsilon}^{y}, q_{\epsilon}^{y}\right)$ process, while each element of $M^{x} \epsilon_{t}^{x}$ is a scalar $\operatorname{ARMA}\left(p_{\epsilon}^{x}, q_{\epsilon}^{x}\right)$ process. These are mutually uncorrelated stationary processes. Multivariate processes complicate the analysis and are less likely to be empirically relevant. When Assumptions A, B and C hold, $\left\{\left(Y_{t}^{\prime}, X_{t}^{\prime}\right)^{\prime}\right\}$ is weakly stationary (see, e.g., Hannan, 1970).

Define the parameters in Model-y and the measurement error processes as follows:

$$
\begin{aligned}
\theta_{A R} & \equiv \operatorname{vec}\left(A_{1}, \ldots, A_{p_{y}}, B_{0}, \ldots, B_{r_{x}}, \Phi_{1}^{y}, \ldots, \Phi_{p_{\epsilon}^{y}}^{y}, \Phi_{1}^{x}, \ldots, \Phi_{p_{\epsilon}^{x}}^{x}\right) \\
\theta_{M A} & \equiv \operatorname{vec}\left(\Sigma_{u}, \Theta_{1}^{y}, \ldots, \Theta_{q_{\epsilon}^{y}}^{y}, \Theta_{1}^{x}, \ldots, \Theta_{q_{\epsilon}^{x}}^{x}, \Sigma_{v}^{y}, \Sigma_{v}^{x}\right) .
\end{aligned}
$$

The objective of the exercise is to identify $\theta_{A R}$ and $\theta_{M A}$.

Consider first identification of the parameters of Model-y in the absence of measurement error. Let $\vec{x}_{t}=\left(x_{t}^{\prime}, \ldots, x_{t-r_{x}}^{\prime}\right)^{\prime}$. If lagged values of $y_{t}$ are absent from Model-y, then $\vec{B}$ is identified if $E\left[u_{t} \vec{x}_{t}^{\prime}\right]=0$ and $E\left[\vec{x}_{t} \vec{x}_{t}^{\prime}\right]$ is full rank, no matter what the dynamics of the unobservables $\left\{u_{t}\right\}$ are. With lagged $y_{t}$ 's in the model, $\vec{A}$ and $\vec{B}$ are identified if for all $t, E\left[u_{t} x_{t-\tau}^{\prime}\right]=0$ for all $\tau \geqslant 0$ and $E\left[u_{t} u_{s}^{\prime}\right]=0$ for all $s<t .{ }^{1}$ Since $u_{t}$ is covariance stationary under Assumption A, time invariance of the second moments permits identification of $\Sigma_{u}$ from the covariance structure of the residuals $A(L) y_{t}-B(L) x_{t}$.

With measurement errors, the model in terms of observables is:

$$
A(L) Y_{t}=B(L) X_{t}+u_{t}+A(L) \epsilon_{t}^{y}-B(L) \epsilon_{t}^{x}
$$

The three terms in $u_{t}+A(L) \epsilon_{t}^{y}-B(L) \epsilon_{t}^{x}$ are not separately observable. Identification of dynamic models in the presence of measurement errors is a nontrivial problem because the composite error term $u_{t}+A(L) \epsilon_{t}^{y}-B(L) \epsilon_{t}^{x}$ will in general fail to be a finite order moving average process, and common factor dynamics cannot be ruled out. This violates classical conditions for identification of VARMA processes derived in Hannan (1969) which require co-primeness even in the absence of exogenous regressors. Furthermore, the composite error is necessarily correlated with $Y_{t}$ and/or $X_{t}$, violating the exogeneity conditions used, for example, in Hannan (1971) and Hannan, Dunsmuir, and Deistler (1980).

\footnotetext{
${ }^{1}$ The exogeneity condition $E\left[u_{t} \vec{x}_{t}^{\prime}\right]=0$ is no longer sufficient as $\vec{y}_{t-1}=\left(y_{t-1}^{\prime}, \ldots, y_{t-p_{y}}^{\prime}\right)^{\prime}$ is a function of all the lagged $x_{t}$ 's and $u_{t}$ 's.
} 


\subsection{Related Literature}

In a seminal paper, Reiersøl (1950) showed that identification in a single regressor static model is possible only if $\left(Y_{t}, X_{t}\right)$ are not jointly normally distributed. However, as discussed in Maravall and Aigner (1977) and Maravall (1979), a static model that is unidentified in the presence of serially uncorrelated measurement errors could be identifiable when the model has a dynamic structure. Intuitively, if $\epsilon_{t}^{y}$ and $\epsilon_{t}^{x}$ are white noise but the model has dynamics, $\vec{A}$ and $\vec{B}$ can, in principle, be identified by suitable choice of instruments such as lags of $Y_{t}$ and $X_{t}$. Aigner, Hsiao, Kapteyn, and Wansbeek (1984, p.1324) pointed out that consistent parameter estimation is also possible with repeated observations on $y_{t}$, or strong a priori information. ${ }^{2}$ Aigner, Hsiao, Kapteyn, and Wansbeek (1984, p.1377) also noted that serially correlated measurement errors will complicate the identification problem, but the econometrics literature on this issue is quite sparse. Mcdonald and Darroch (1983) consider single equation estimation of $\vec{A}$ and $\vec{B}$ with correlated measurement errors but did not consider separate identification of the equation error and the measurement noise.

There is, however, a reasonably large literature in control theory studying the identification of noise corrupted "multiple input-multiple output" (MIMO) models; see Söderström (2007) for a survey. By defining a new latent endogenous variable $y_{t}^{*} \equiv y_{t}-A(L)^{-1} u_{t}$, Model-y can be written as a MIMO model

$$
y_{t}^{*}=B^{*}(L) x_{t}
$$

where $B^{*}(L) \equiv A(L)^{-1} B(L)$. In a noise-corrupted analysis, $x_{t}$ is referred to as the input vector and $y_{t}$ is the output vector. The observed variables are $X_{t}=x_{t}+\epsilon_{t}^{x}$ and $Y_{t}=y_{t}^{*}+\epsilon_{t}^{y *}$, where the new measurement error $\epsilon_{t}^{y *} \equiv A(L)^{-1} u_{t}+\epsilon_{t}^{y}$ amalgamates the shocks $u_{t}$ and the observational errors $\epsilon_{t}^{y}$; see Solo (1986).

While a specification with no error term on the right hand side is uncommon in econometric analysis, $y_{t}$ has a MIMO representation and results from that literature are relevant. The key result, shown for the case of a single input and single output (also known as SISO systems), is given in Anderson and Deistler (1984). They show that the transfer function $B^{*}(L)$ (or the Wold representation) of such systems is impossible to identify nonparametrically. This "essential nonidentifiability" holds even if the system is known to be causal. Identification is possible only if some structure is put on the measurement errors. One alternative is to treat $\epsilon_{t}^{x}$ as a dynamic factor, and $B(L)$ are the factor loadings. A second alternative is to parametrically specify the covariance structure of the residuals. Anderson and Deistler (1984) assume that the input and the

\footnotetext{
${ }^{2}$ Erickson and Whited (2002) used higher order moments, while Willassen (1977) used the joint distribution of the observables for identification. Hsiao (1979) studies identification of dynamic simultaneous equations models from the second moment properties of the data when the measurement errors are white noise.
} 
measurement errors are ARMA processes and obtain conditions for identification from the second moments of the data without restricting the lag orders a priori. The multivariate extension to stationary and causal MIMO-EIV systems is considered in Nowak (1992, 1993).

The common feature of these results is that they all require a parametric model for $x_{t}$. For instance, Nowak $(1992,1993)$ assume that $x_{t}$ are VARMA processes. Parametric identification when the data are correctly observed dates back to Koopmans (1953) and Fisher (1963). Measurement errors impose the additional requirement that the model for $x_{t}$ has to be specified. This is restrictive if $x_{t}$ 's are thought to be determined outside of the system defined by Model-y.

An alternative to MIMO models are state space systems that explicitly allow for measurement noise. Sargent (1989) shows how the Kalman filter can be used when the variables in a dynamic model are subject to autoregressive measurement errors. But much like the MIMO models, the process for every variable in the model needs to be completely specified. There are in fact relatively few identification results for which the exogenous variables are not explicitly modeled. Solo (1986) considers an ARMAX model without imposing assumptions on the exogenous variable and shows that the identification conditions agree with those derived by Maravall (1979) with parametric assumptions on the exogenous process. Our approach is similar in spirit to Solo (1986) with several considerations in mind.

First, if the primitive shocks of a structural VAR were to be subsequently recovered from the reduced form errors $u_{t}$, then $u_{t}$ needs to be separated from the measurement noise $\epsilon_{t}^{y}$. Considering the composite error $\epsilon_{t}^{y *}$ as in MIMO systems is not enough. Second, we restrict attention to VARX models because VARMAX models are rarely used in economic applications. Requiring $u_{t}$ to be white noise rules out observational equivalence that may arise from common factors between $A(L)$ and $u_{t}$. Third, we do not specify the distribution of the measurement errors or of the exogenous variables. The only restriction is that the measurement errors are scalar ARMA processes. Parametric assumptions on the measurement errors are necessary in view of the result of Anderson and Deistler (1984). One can think of this limited information approach as a semi-parametric setup in which the distribution of the measurement errors and the exogenous variables are the nonparametric part of the model.

\section{Order Condition for Mismeasured VARX Models}

Our point of departure is that when the measurement errors are serially correlated, the dynamic representation for $Y_{t}$ will, in general, be different from that for $y_{t}$ because $Y_{t}$ is the sum of two latent dynamic processes. In a univariate setting, Granger and Morris (1976) showed that if $y_{t}$ is $\operatorname{ARMA}\left(p_{y}, q_{y}\right)$ and $\epsilon_{t}^{y}$ is $\operatorname{ARMA}\left(p_{\epsilon}^{y}, q_{\epsilon}^{y}\right)$, then $Y_{t}=y_{t}+\epsilon_{t}^{y}$ is $\operatorname{ARMA}(p, q)$ where $p \leqslant p_{y}+p_{\epsilon}^{y}$ and 
$q \leqslant \max \left\{p_{y}+q_{\epsilon}^{y}, p_{\epsilon}^{y}+q_{y}\right\} .{ }^{3}$ The following lemma provides a general result for vector processes.

Lemma 1 Suppose Assumptions $A, B$ and $C$ hold. Then the observed data can be represented as:

$$
\mathcal{A}(L) Y_{t}=\mathcal{B}(L) X_{t}+U_{t}
$$

where $\mathcal{A}(L)$ and $\mathcal{B}(L)$ are polynomials of orders $p_{Y}$ and $r_{X}$ whose coefficients only depend on $\theta_{A R}$, $U_{t}$ is a $V M A\left(q_{U}\right)$ error process that depends on both $\theta_{A R}$ and $\theta_{M A}$, and

$$
\begin{aligned}
& p_{Y}=p_{y}+\bar{p} \\
& q_{U} \leqslant \bar{p}+\max \left\{0,\left[p_{y}+q_{\epsilon}^{y}-p_{\epsilon}^{y}\right] \cdot 1\left(n_{\epsilon}^{y}>0\right),\left[r_{x}+q_{\epsilon}^{x}-p_{\epsilon}^{x}\right] \cdot 1\left(n_{\epsilon}^{x}>0\right)\right\} \\
& r_{X}=r_{x}+\bar{p},
\end{aligned}
$$

for some $\bar{p} \leqslant n_{\epsilon}^{y} p_{\epsilon}^{y}+n_{\epsilon}^{x} p_{\epsilon}^{x}$. Moreover, $E\left(U_{t}\right)=0, E\left(U_{t} Y_{t-\tau}^{\prime}\right)=0$ for all $\tau>\tau_{Y}$, and $E\left(U_{t} X_{t-\tau}^{\prime}\right)=0$ for all $\tau>\tau_{X}$, where

$$
\begin{gathered}
\tau_{Y} \leqslant \bar{p}+\max \left\{0,\left[p_{y}+q_{\epsilon}^{y}-p_{\epsilon}^{y}\right] \cdot 1\left(n_{\epsilon}^{y}>0\right)\right\} \\
\tau_{X} \leqslant \bar{p}+\max \left\{0,\left[r_{x}+q_{\epsilon}^{x}-p_{\epsilon}^{x}\right] \cdot 1\left(n_{\epsilon}^{x}>0\right)\right\} .
\end{gathered}
$$

Lemma 1 shows that Model-Y has richer dynamics than Model-y. The autoregressive part of Model-Y is of order $p_{Y} \geqslant p_{y}$. Furthermore, $Y_{t}$ has a moving average component even if none is present in $y_{t}$ and $\Gamma_{U}(j) \equiv E\left(U_{t} U_{t-j}^{\prime}\right) \neq 0$ for all $j=1, \ldots, q_{U}$. In consequence, the usual orthogonality conditions $E\left(U_{t} Y_{t-\tau}^{\prime}\right)=0$ and $E\left(U_{t} X_{t-\tau}\right)=0$ no longer hold for all $\tau>0$. Instead, $E\left(U_{t} Y_{t-\tau}^{\prime}\right)=0$ only for $\tau>\tau_{Y}$, and $E\left(U_{t} X_{t-\tau}^{\prime}\right)=0$ only for $\tau>\tau_{X}$. The composite error $U_{t}$ is a moving average process of order

$$
q_{U}=\max \left[\tau_{Y}, \tau_{X}\right]
$$

In general, $\tau_{Y} \neq \tau_{X}$ because $p_{y}$ and $r_{x}$ may differ, and so can the dynamics of the measurement errors in $Y_{t}$ and $X_{t}$. Even though under Assumption $\mathrm{C}$ the measurement errors are mutually uncorrelated ARMA processes, $U_{t}$ will follow a vector MA process whenever one of the polynomials $A(L)$ and $B(L)$ is not diagonal.

Although $p_{Y}, r_{X}$ and $q_{U}$ can be identified from the data, point identification of $p_{y}, r_{x}, p_{\epsilon}^{y}, q_{\epsilon}^{y}, p_{\epsilon}^{x}, q_{\epsilon}^{x}$ necessitates a priori restrictions. For this reason, Assumptions $\mathrm{B}$ and $\mathrm{C}$ treat these parameters as fixed. Interestingly, Lemma 1 imply that the true lags $p_{y}$ and $r_{x}$ are always set identified in the sense that $0 \leqslant p_{y} \leqslant p_{Y}$ and $0 \leqslant r_{x} \leqslant r_{X}$.

\footnotetext{
${ }^{3}$ For example, if $y_{t}$ and $\epsilon_{t}^{y}$ are $A R(1)$ with different coefficients, then $\left\{Y_{t}\right\}$ is $A R M A(2,1)$.
} 
From the data $Y_{t}$ and $X_{t}$ and the assumed lag orders, the most we can uncover are the parameters in Model-Y:

$$
\begin{aligned}
\pi_{A R} & \equiv \operatorname{vec}\left(\mathcal{A}_{1}, \ldots, \mathcal{A}_{p_{Y}}, \mathcal{B}_{0}, \ldots, \mathcal{B}_{r_{X}}\right) \\
\pi_{M A} & \equiv \operatorname{vec}\left(\Gamma_{U}(0), \ldots, \Gamma_{U}\left(q_{U}\right)\right) .
\end{aligned}
$$

The question is whether knowing the parameters $\pi^{\prime} \equiv\left(\pi_{A R}^{\prime}, \pi_{M A}^{\prime}\right)$ enables identification of the parameters $\theta^{\prime} \equiv\left(\theta_{A R}^{\prime}, \theta_{M A}^{\prime}\right)$ without fully specifying the data generating process for $x_{t}$. A key to our order condition for identification is to exploit the specific structure implied by Lemma 1 :

$$
\begin{aligned}
\pi_{A R} & =\pi_{A R}\left(\theta_{A R}\right) \\
\pi_{M A} & =\pi_{M A}\left(\theta_{A R}, \theta_{M A}\right)
\end{aligned}
$$

This observation leads to the following order conditions.

Proposition 1 Suppose Assumptions A, B and C hold. Assume that $\pi(\theta)$ is continuously differentiable and that the rank of $\frac{\partial \pi}{\partial \theta}$ remains constant in a neighborhood of $\theta$. The necessary order conditions for local identification of $\theta_{A R}$ and $\theta_{M A}$ jointly are,

$$
\begin{array}{ll}
A R: & \operatorname{dim}\left(\theta_{A R}\right) \leqslant \operatorname{dim}\left(\pi_{A R}\right)+\operatorname{dim}\left(\pi_{M A}\right)-\operatorname{dim}\left(\theta_{M A}\right) \\
M A: & \operatorname{dim}\left(\theta_{M A}\right) \leqslant \operatorname{dim}\left(\pi_{M A}\right) .
\end{array}
$$

The results exploit the triangular structure of the problem and are new to the literature. Since $\theta_{M A}$ appears in $\pi_{M A}$ and not in $\pi_{A R}, \theta_{M A}$ can only be identified from $\pi_{M A}$ and the MA condition follows. If $\theta_{M A}$ is overidentified, the additional information can be used to identify $\theta_{A R}$, leading to the AR condition. These order restrictions are more stringent than just requiring that $\operatorname{dim}\left(\theta_{A R}\right)+$ $\operatorname{dim}\left(\theta_{M A}\right) \leqslant \operatorname{dim}\left(\pi_{A R}\right)+\operatorname{dim}\left(\pi_{M A}\right)$. Proposition 1 can be made more explicit upon substituting in the dimensions of the parameters:

$$
\begin{aligned}
\operatorname{dim}\left(\theta_{A R}\right) & =n_{y}^{2} \cdot p_{y}+n_{y} \cdot n_{x} \cdot\left(r_{x}+1\right)+n_{\epsilon}^{y} \cdot p_{\epsilon}^{y}+n_{\epsilon}^{x} \cdot p_{\epsilon}^{x} \\
\operatorname{dim}\left(\theta_{M A}\right) & =\frac{n_{y}\left(n_{y}+1\right)}{2}+n_{\epsilon}^{y}+n_{\epsilon}^{y} \cdot q_{\epsilon}^{y}+n_{\epsilon}^{x}+n_{\epsilon}^{x} \cdot q_{\epsilon}^{x} \\
\operatorname{dim}\left(\pi_{A R}\right) & =n_{y}^{2} \cdot p_{Y}+n_{y} \cdot n_{x} \cdot\left(r_{X}+1\right) \\
\operatorname{dim}\left(\pi_{M A}\right) & =\frac{n_{y}\left(n_{y}+1\right)}{2}+n_{y}^{2} \cdot q_{U} .
\end{aligned}
$$

The AR order condition holds whenever the following two conditions are satisfied:

$$
n_{y}^{2}\left(p_{Y}-p_{y}\right) \geqslant n_{\epsilon}^{y} p_{\epsilon}^{y} \quad \text { and } \quad n_{y} n_{x}\left(r_{X}-r_{x}\right) \geqslant n_{x}^{\epsilon} p_{\epsilon}^{x}
$$


Although simple instrumental variables arguments can be used to establish that $\mathcal{A}_{j}$ and $\mathcal{B}_{j}$ are identified, $\mathcal{A}_{j} \neq A_{j}$ and $\mathcal{B}_{k} \neq B_{k}$ in the presence of measurement errors. The above conditions ensure that the dynamics of Model-Y are sufficiently richer than those of Model-y to disentangle the effects of lagged $y_{t}$ and $x_{t}$ from the measurement noise. The required difference in dynamics depends on the number of variables measured with errors. The conditions are however stronger than necessary.

The MA order condition requires that

$$
\begin{aligned}
n_{\epsilon}^{y}\left(q_{\epsilon}^{y}+1\right) & +n_{\epsilon}^{x}\left(q_{\epsilon}^{x}+1\right) \leqslant \\
& n_{y}^{2}\left[n_{\epsilon}^{y} p_{\epsilon}^{y}+n_{\epsilon}^{x} p_{\epsilon}^{x}+\max \left\{0,\left[p_{y}+q_{\epsilon}^{y}-p_{\epsilon}^{y}\right] \cdot 1\left(n_{\epsilon}^{y}>0\right),\left[r_{x}+q_{\epsilon}^{x}-p_{\epsilon}^{x}\right] \cdot 1\left(n_{\epsilon}^{x}>0\right)\right\}\right],
\end{aligned}
$$

which evidently depends on the lag structure of the model (as given by $p_{y}$ and $r_{x}$ ), and the dynamics of the measurement errors (as given by $p_{\epsilon}^{y}, q_{\epsilon}^{y}, p_{\epsilon}^{x}, q_{\epsilon}^{x}$ ). If the condition fails, then $\theta_{M A}$ cannot be recovered from the variance and the $q_{U}$ autocovariances of $U_{t}$ collected in $\pi_{M A}$. As our subsequent examples will show, the MA order condition is more likely to be violated than the AR condition even in simple models. It is therefore possible that $\operatorname{dim}\left(\theta_{A R}\right)+\operatorname{dim}\left(\theta_{M A}\right) \leqslant \operatorname{dim}\left(\pi_{A R}\right)+\operatorname{dim}\left(\pi_{M A}\right)$ and yet $\operatorname{dim}\left(\theta_{M A}\right)>\operatorname{dim}\left(\pi_{M A}\right)$.

The results of Proposition 1 have implications for identification of the structural parameters in the simultaneous equations system:

$$
\mathbb{A}_{0} y_{t}=\mathbb{A}_{1} y_{t-1}+\ldots+\mathbb{A}_{p_{y}} y_{t-p_{y}}+\mathbb{B}_{0} x_{t}+\ldots+\mathbb{B}_{r_{x}} x_{t-r_{x}}+\mathbb{U}_{t}
$$

with $\mathbb{A}_{0} \neq I$, and whose reduced form is Model-y. Hsiao (1979) develops exclusion restrictions for the case of white noise measurement errors. Because identification of Model-y is necessary for identification of $\left(\mathbb{A}_{1}, \ldots, \mathbb{A}_{p_{y}}\right),\left(\mathbb{B}_{0}, \ldots, \mathbb{B}_{r_{x}}\right)$ and $\Sigma_{\mathbb{U}}$, the order conditions presented above are also necessary for identification of the structural parameters.

Proposition 2 Suppose Assumptions $A, B$ and $C$ hold. Assume that $\pi(\theta)$ is continuously differentiable, and that the ranks of $\frac{\partial \pi}{\partial \theta}, \frac{\partial \pi}{\partial \theta_{A R}}$ and $\frac{\partial \pi}{\partial \theta_{M A}}$ remain constant in a neighborhood of $\theta$. The necessary order conditions for local identification of $\theta_{A R}$ alone and of $\theta_{M A}$ alone are, respectively,

$$
\begin{array}{ll}
A R: & \operatorname{dim}\left(\theta_{A R}\right) \leqslant \operatorname{dim}\left(\pi_{A R}\right)+\operatorname{dim}\left(\pi_{M A}\right) \\
M A: & \operatorname{dim}\left(\theta_{M A}\right) \leqslant \operatorname{dim}\left(\pi_{M A}\right) .
\end{array}
$$

If one is interested in $\theta_{M A}$ alone, the necessary order condition for identification is simply $\operatorname{dim}\left(\theta_{M A}\right) \leqslant \operatorname{dim}\left(\pi_{M A}\right)$. This is hardly surprising since $\theta_{M A}$ can only be recovered from $\pi_{M A}$. However, $\theta_{A R}$ can potentially be recovered from all information available. This leads to the weaker order condition: $\operatorname{dim}\left(\theta_{A R}\right) \leqslant \operatorname{dim}\left(\pi_{A R}\right)+\operatorname{dim}\left(\pi_{M A}\right)$. 


\subsection{Two Special Cases: ARX Models and VAR}

This subsection studies the implications of Proposition 1 for important special cases: scalar ARX models and pure VARs. We focus on the joint identification of $\theta_{A R}$ and $\theta_{M A}$.

Case 1: $\operatorname{ARX}\left(p_{y}, r_{x}\right)$ with $n_{x}=1, n_{\epsilon}^{y}=0, n_{\epsilon}^{x}=1$. When $x_{t}$ is scalar, Model-y is

$$
y_{t}=A_{1} y_{t-1}+\ldots+A_{p_{y}} y_{t-p_{y}}+B_{0} x_{t}+\ldots+B_{r_{x}} x_{t-r_{x}}+u_{t}, \quad u_{t} \sim W N\left(0, \Sigma_{u}\right) .
$$

If $y_{t}$ is correctly measured but $x_{t}$ is measured with errors, Model-Y is $A R M A\left(p_{Y}, q_{U}, r_{X}\right)$ with $p_{Y}=p_{y}+p_{\epsilon}^{x}, q_{U}=p_{\epsilon}^{x}+\max \left\{0, r_{x}+q_{\epsilon}^{x}-p_{\epsilon}^{x}\right\}$, and $r_{X}=r_{x}+p_{\epsilon}^{x}{ }^{4}$ The model is quite commonly used as latent variables such as expected inflation, permanent income, wealth, marginal $Q$ play important roles in economic analysis. For example, the Phillips curve has $x_{t}$ being the output gap and $y_{t}$ being inflation. As the output gap is latent, its proxy can be thought of as a mismeasured variant of $x_{t}$. If this proxy is constructed by filtering an observed variable, it is likely that $\epsilon_{t}^{x}$ will be serially correlated.

Applying Lemma 1, we have $\operatorname{dim}\left(\theta_{A R}\right)=p_{y}+\left(r_{x}+1\right)+p_{\epsilon}^{x}, \operatorname{dim}\left(\theta_{M A}\right)=2+q_{\epsilon}^{x}, \operatorname{dim}\left(\pi_{A R}\right)=$ $p_{y}+\left(r_{x}+1\right)+2 p_{\epsilon}^{x}$, and $\operatorname{dim}\left(\pi_{M A}\right)=1+p_{\epsilon}^{x}+\max \left\{0, r_{x}+q_{\epsilon}^{x}-p_{\epsilon}^{x}\right\}$. Consider the following cases:

$$
\operatorname{ARX}\left(p_{y}, r_{x}\right) \text { with } n_{\epsilon}^{y}=0 \text {. }
$$

\begin{tabular}{|c|c|c|c|c|c|c|c|c|c|c|}
\hline & \multicolumn{3}{|c|}{$p_{y}=0, r_{x}=0$} & \multicolumn{2}{|c|}{ Order Condition } & \multicolumn{3}{|c|}{$p_{y}=1, r_{x}=0$} & \multicolumn{2}{|c|}{ Order Condition } \\
\hline$\epsilon_{t}^{x}$ & $p_{Y}$ & $q_{U}$ & $r_{X}$ & $A R$ & $M A$ & $p_{Y}$ & $q_{U}$ & $r_{X}$ & $A R$ & $M A$ \\
\hline $\mathrm{WN}$ & 0 & 0 & 0 & pass & fail & 1 & 0 & 0 & pass & fail \\
\hline $\mathrm{MA}(1)$ & 0 & 1 & 0 & pass & fail & 1 & 1 & 0 & pass & fail \\
\hline $\operatorname{AR}(1)$ & 1 & 1 & 1 & pass & pass & 2 & 1 & 1 & pass & pass \\
\hline $\operatorname{ARMA}(1,1)$ & 1 & 1 & 1 & pass & fail & 2 & 1 & 1 & pass & fail \\
\hline & $p_{y}$ & $2, r$ & $=0$ & Orde & ondition & $p_{y}$ & 1, & $=1$ & Orde & ondition \\
\hline$\epsilon_{t}^{x}$ & $p_{Y}$ & $q_{U}$ & $r_{X}$ & $A R$ & $M A$ & $p_{Y}$ & $q_{U}$ & $r_{X}$ & $A R$ & $M A$ \\
\hline WN & 2 & 0 & 0 & pass & fail & 1 & 1 & 1 & pass & pass \\
\hline $\mathrm{MA}(1)$ & 2 & 0 & 0 & pass & fail & 1 & 2 & 1 & pass & pass \\
\hline $\operatorname{AR}(1)$ & 3 & 1 & 1 & pass & pass & 2 & 1 & 2 & pass & pass \\
\hline $\operatorname{ARMA}(1,1)$ & 3 & 1 & 1 & pass & fail & 2 & 2 & 2 & pass & pass \\
\hline
\end{tabular}

When there are no lags of the dependent variable (hence $p_{y}=0$ ) and $r_{x}=0$, Model-y is static. The autoregressive dynamics in Model-Y alone are not sufficient for identification since the residual autocovariances need to provide information for recovering the components of $\theta_{M A}$. The MA order condition fails except when $\epsilon_{t}^{x}$ is $\operatorname{AR}(1)$. The condition also fails if $p_{y}=1$ or $p_{y}=2$, with $r_{x}=0$

\footnotetext{
${ }^{4}$ When all the variables in Model-y are scalar, $n_{\epsilon}^{y}=0$ and $B(L) \neq \Phi^{x}(L)$, then the results of Lemma 1 hold with equalities in place of (upper) inequalities.
} 
because there are fewer non-zero lagged autocovariances of $U_{t}$ than what is needed to recover all MA parameters. Identification is more likely to obtain when Model-y is dynamic. As indicated from the table, the MA order condition holds for any value of $p_{y}$ whenever $r_{x} \geqslant 1$. The identification conditions can be formalized as follows.

Corollary 1 Consider an $A R X\left(p_{y}, r_{x}\right)$ model in which the scalar variable $x_{t}$ is observed with error, and let all the assumptions of Proposition 1 hold. The AR order condition for joint local identification of $\theta_{A R}$ and $\theta_{M A}$ always holds; the MA order condition for joint local identification of $\theta_{A R}$ and $\theta_{M A}$ requires $\max \left\{p_{\epsilon}^{x}-q_{\epsilon}^{x}, r_{x}\right\} \geqslant 1$.

The corollary makes clear that the AR order condition always holds, but the necessary condition for identifying $\theta_{M A}$ can fail. More precisely, $\operatorname{dim}\left(\pi_{A R}\right)+\operatorname{dim}\left(\pi_{M A}\right)$ can exceed $\operatorname{dim}\left(\theta_{A R}\right)+\operatorname{dim}\left(\theta_{M A}\right)$ and yet the order condition for $\theta_{M A}$ fails. In the case of an $\operatorname{ARX}(1,0)$ model with $\operatorname{ARMA}(1,1)$ measurement error for example, $\operatorname{dim}(\pi)=\operatorname{dim}(\theta)=6$, but $\operatorname{dim}\left(\pi_{M A}\right)-\operatorname{dim}\left(\theta_{M A}\right)=-1$. Although the total number of parameters in Model-Y and Model-y are equal, this total number is uninformative and misleading about the identifiability of Model-y. The issue is that $\pi_{A R}$ tends to overidentify $\theta_{A R}$, but the overidentifying conditions are of no help in identifying $\theta_{M A}$. For this reason, the MA and AR order conditions need to be considered jointly.

Case 2: $\operatorname{ARX}\left(p_{y}, r_{x}\right)$ with $n_{x}=n_{\epsilon}^{y}=n_{\epsilon}^{x}=1$. When in $x_{t}$ and $y_{t}$ are both mismeasured, the dynamic structure of $y_{t}$ and that of $\epsilon_{t}^{y}$ will both play a role in identification of $\theta_{M A}$. This is illustrated for the case when $\epsilon_{t}^{y} \sim A R(1)$.

\begin{tabular}{l|lllll|lllll}
\multicolumn{10}{c}{$A R X\left(p_{y}, r_{x}\right)$} & with $\left(p_{\epsilon}^{y}, q_{\epsilon}^{y}\right)=(1,0)$. \\
\hline & $p_{y}=2, r_{x}=0$ & Order Condition & $p_{y}=0, r_{x}=2$ & Order Condition \\
\hline$\epsilon_{t}^{x}$ & $p_{Y}$ & $q_{U}$ & $r_{X}$ & $A R$ & $M A$ & $p_{Y}$ & $q_{U}$ & $r_{X}$ & $A R$ & $M A$ \\
\hline WN & 3 & 2 & 1 & pass & pass & 1 & 3 & 3 & pass & pass \\
MA(1) & 3 & 2 & 1 & pass & fail & 1 & 4 & 3 & pass & pass \\
AR(1) & 4 & 3 & 2 & pass & pass & 2 & 4 & 4 & pass & pass \\
ARMA $(1,1)$ & 4 & 3 & 2 & pass & pass & 2 & 4 & 4 & pass & pass \\
\hline
\end{tabular}

Corollary 2 Consider an $A R X\left(p_{y}, r_{x}\right)$ model in which the scalar variables $y_{t}$ and $x_{t}$ are observed with errors and $\epsilon_{t}^{y}$ is an AR(1) process, and let all the assumptions of Proposition 1 hold. The AR order condition for joint local identification of $\theta_{A R}$ and $\theta_{M A}$ always holds; the MA order condition for joint local identification of $\theta_{A R}$ and $\theta_{M A}$ requires $\max \left\{p_{\epsilon}^{x}-q_{\epsilon}^{x}, p_{y}-1+p_{\epsilon}^{x}-q_{\epsilon}^{x}, r_{x}\right\} \geqslant 1$.

The order condition for identifying $\theta_{M A}$ holds with white noise but not with MA(1) errors in $x_{t}$. This is because $q_{U}$ is the same in both cases, but the lags of $U_{t}$ provide no additional information to 
identify the $\mathrm{MA}(1)$ parameter in the measurement error process. In such a case, the order condition requires either that $r_{x}$ be greater than one, or that the measurement error be an $\operatorname{ARMA}(1,1)$.

Case 3: $\operatorname{VAR}\left(p_{y}\right) \quad$ Vector regressions are widely used in macroeconomic analysis. In the presence of serially correlated measurement errors, a $\operatorname{VAR}\left(p_{y}\right)$ model becomes a $\operatorname{VARMA}\left(p_{Y}, q_{U}\right)$.

Corollary 3 Consider a $\operatorname{VAR}\left(p_{y}\right)$ process in which some (possibly all) components of the $n_{y}$ vector $y_{t}$ are observed with error, and let all the assumptions of Proposition 1 hold. The AR order condition for joint local identification of $\theta_{A R}$ and $\theta_{M A}$ is always satisfied; the MA order condition for joint local identification of $\theta_{A R}$ and $\theta_{M A}$ requires that

$$
\max \left\{\frac{n_{y}^{2}}{n_{\epsilon}^{y}} p_{y}+\left(\frac{n_{y}^{2}}{n_{\epsilon}^{y}}-1\right) q_{\epsilon}^{y}+\frac{n_{y}^{2}}{n_{\epsilon}^{y}}\left(n_{\epsilon}^{y}-1\right) p_{\epsilon}^{y}, n_{y}^{2} p_{\epsilon}^{y}-q_{\epsilon}^{y}\right\} \geqslant 1 .
$$

Note that since $n_{\epsilon}^{y} \leqslant n_{y}$, the first term inside the max operator is always greater than or equal to $p_{y}$. Thus, if $p_{y} \geqslant 1$ the order condition for identification of $\theta_{M A}$ always holds. This comes from the fact that our measurement errors are univariate ARMA and not VARMA processes. Thus, while $\operatorname{dim}\left(\pi_{M A}\right)=O\left(n_{y}^{2}\right)$, the number of measurement error parameters is $O\left(n_{\epsilon}^{y}\right)$. The order conditions for $\operatorname{VAR}\left(p_{y}\right)$ models with $p_{y} \geqslant 1$ are thus satisfied for all $n_{y}$. Interestingly, the result implies that seemingly unrelated systems might make it possible to identify parameters that are otherwise non-identifiable in a single equation setup. In the case of an $A R\left(p_{y}\right)$ model (i.e. $n_{y}=1$ ), the order condition for identifying $\theta_{M A}$ simplifies to $\max \left\{p_{y}, p_{y}^{\epsilon}-q_{\epsilon}^{y}\right\} \geqslant 1$.

\section{Rank Condition for Mismeasured VARX Models}

The preceding analysis has focused on conditions that are necessary for identifying Model-y. The order conditions are not sufficient because Model-Y may not be identified. Showing that Model-Y is identifiable is not trivial for two reasons. First, the covariates $X_{t}$ are no longer strongly exogenous. Second, Model-Y has a VARMAX structure that is susceptible to the presence of common factors that render the parameters in Model-Y non-identifiable. However, deriving a canonical form for Model-Y that is free of common factors is often not a trivial task (see, e.g., Hannan, 1971).

The following lemma gives sufficient conditions for identification of Model-Y that do not require

canonical forms. Let $\Gamma_{Y Y}(j) \equiv E\left(Y_{t} Y_{t-j}^{\prime}\right), \Gamma_{X X}(j) \equiv E\left(X_{t} X_{t-j}^{\prime}\right), \Gamma_{X Y}(j) \equiv E\left(X_{t} Y_{t-j}^{\prime}\right)$ and $\Gamma_{Y X}(j) \equiv E\left(Y_{t} X_{t-j}^{\prime}\right)$ be the auto-covariances and cross-covariances of $\left\{Y_{t}\right\}$ and $\left\{X_{t}\right\}$. Define

$$
\begin{array}{ll}
H_{Y Y} \equiv\left(\Gamma_{Y Y}\left(j-i+\tau_{Y}\right)\right)_{1 \leqslant i, j \leqslant p_{Y}} & H_{Y X} \equiv\left(\Gamma_{Y X}\left(j-i+\tau_{X}\right)\right)_{1 \leqslant i \leqslant p_{Y}, 1 \leqslant j \leqslant r_{X}+1} \\
H_{X Y} \equiv\left(\Gamma_{X Y}\left(j-i+\tau_{Y}+1\right)\right)_{1 \leqslant i \leqslant r_{X}+1,1 \leqslant j \leqslant p_{Y}} & H_{X X} \equiv\left(\Gamma_{X X}\left(j-i+\tau_{X}+1\right)\right)_{1 \leqslant i, j \leqslant r_{X}+1}
\end{array}
$$


Lemma 2 Suppose Assumptions $A, B$ and $C$ hold. Then, the parameters $\pi_{A R}$ and $\pi_{M A}$ of Model- $Y$ are jointly globally identifiable if and only if

$$
\operatorname{rank}\left(\begin{array}{ll}
H_{Y Y} & H_{Y X} \\
H_{X Y} & H_{X X}
\end{array}\right)=n_{y} p_{Y}+n_{x}\left(r_{X}+1\right)
$$

The key insight of Lemma 2 is that the orthogonality restrictions $E\left(U_{t} Y_{t-\tau}^{\prime}\right)=0$ for $\tau>\tau_{Y}$ and $E\left(U_{t} X_{t-\tau}^{\prime}\right)=0$ for $\tau>\tau_{X}$ put enough structure to guarantee identification of Model-Y. The result requires, however, that there be enough information in the auto-covariances and cross-covariances of $\left\{Y_{t}\right\}$ and $\left\{X_{t}\right\}$ to satisfy the rank condition. Since Model-Y is linear in $\pi_{A R}$, the rank condition is both necessary and sufficient, and the identification result is global.

As an example, consider an $\operatorname{ARX}(1,0)$ model in which both $y_{t}$ and $x_{t}$ are subject to white noise measurement errors. In that case, $Y_{t}=A_{1} Y_{t-1}+B_{0} X_{t}+u_{t}+v_{t}^{y}-A_{1} v_{t-1}^{y}-B_{0} v_{t}^{x}$ is an $\operatorname{ARMAX}(1,1,0)$ with $\tau_{Y}=1$ and $\tau_{X}=0$. Now if the latent covariates $x_{t}$ are themselves a white noise process, the matrix in Lemma 2 reduces to:

$$
\left(\begin{array}{ll}
\Gamma_{Y Y}(1) & \Gamma_{Y X}(0) \\
\Gamma_{X Y}(2) & \Gamma_{X X}(1)
\end{array}\right)=\left(\begin{array}{cc}
\Gamma_{y y}(1) & \Gamma_{y x}(0) \\
0 & 0
\end{array}\right),
$$

which clearly fails to satisfy the rank condition. Thus, we recover the well-known result that identification of EIV systems fails when the covariates are white noise. Lemma 2 is an extension of the results in Solo (1986) and Hsiao (1979) to multivariate systems with independent ARMA measurement errors.

Once the parameters of Model-Y are identified, we can proceed with the identification of Modely. Sufficient rank conditions for $\theta_{A R}$ and $\theta_{M A}$ to be jointly locally identified from $\pi_{A R}$ and $\pi_{M A}$ are summarized in the following proposition.

Proposition 3 Suppose Assumptions A, B and C hold. Assume in addition that $\pi(\theta)$ is continuously differentiable and that the rank condition of Lemma 2 holds. Then, sufficient rank conditions for local identification of $\theta_{A R}$ and $\theta_{M A}$ jointly are,

$$
\begin{array}{ll}
A R: & \operatorname{rank}\left(\frac{\partial \pi_{A R}\left(\theta_{A R}\right)}{\partial \theta_{A R}}\right)=\operatorname{dim}\left(\theta_{A R}\right) \\
M A: & \operatorname{rank}\left(\frac{\partial \pi_{M A}\left(\theta_{A R}, \theta_{M A}\right)}{\partial \theta_{M A}}\right)=\operatorname{dim}\left(\theta_{M A}\right) .
\end{array}
$$

The result of Proposition 3 exploits the triangular structure of the system. The practical implication of Proposition 3 is that identification of the autoregressive parameters precedes identification of the variances and moving average parameters of the measurement error process. Even if the AR rank condition is satisfied, the MA rank for condition may fail.

The following result provides separate rank conditions for partial identification of either $\theta_{A R}$ or $\theta_{M A}$ alone. 
Proposition 4 Suppose Assumptions $A, B$ and $C$ hold. Assume in addition that $\pi(\theta)$ is continuously differentiable and that the rank condition of Lemma 2 holds. Then, sufficient rank conditions for local identification of $\theta_{A R}$ alone and of $\theta_{M A}$ alone are, respectively,

$$
\begin{aligned}
& A R: \quad \operatorname{rank}\left(\frac{\partial \pi_{A R}\left(\theta_{A R}\right)}{\partial \theta_{A R}}\right)=\operatorname{dim}\left(\theta_{A R}\right) \\
& M A: \quad \operatorname{rank}\left(\frac{\partial \pi\left(\theta_{A R}, \theta_{M A}\right)}{\partial \theta}\right)=\operatorname{rank}\left(\frac{\partial \pi\left(\theta_{A R}, \theta_{M A}\right)}{\partial \theta_{A R}}\right)+\operatorname{dim}\left(\theta_{M A}\right) .
\end{aligned}
$$

Since $\pi_{A R}$ is a function of $\theta_{A R}$ alone, same rank condition as in Proposition 3 obtains. The condition is sufficient to identify $\theta_{A R}$ irrespective of $\theta_{M A}$. Thus, it is possible that $\theta_{A R}$ is identified and yet $\theta_{M A}$ fails to be so. The rank condition for $\theta_{M A}$ now takes into account that $\theta_{A R}$ may no longer be identified.

\subsection{Example}

There are several ways in which the rank conditions may be checked. In simple models in which the mapping from $\theta$ to $\pi$ is easy to compute analytically, evaluating the rank conditions is straightforward, though formal testing is not. In general, however, numerical approaches may be easier to implement. To illustrate how the rank condition presented in Proposition 3 is to be used, consider the model $y_{t}=A_{1} y_{t-1}+B_{0} x_{t}+B_{1} x_{t-1}+u_{t}$ with $u_{t} \sim W N\left(0, \sigma_{u}^{2}\right)$. While $y_{t}$ is correctly measured, we only observe $X_{t}=x_{t}+\epsilon_{t}$ where $\epsilon_{t}$ is $\operatorname{ARMA}(1,1), \epsilon_{t}=\Phi \epsilon_{t-1}+v_{t}+\Theta v_{t-1}, v_{t} \sim W N\left(0, \sigma_{v}^{2}\right)$. The parameters $\theta^{\prime}=\left(\theta_{A R}^{\prime}, \theta_{M A}^{\prime}\right)$ of Model-y are:

$$
\theta_{A R} \equiv\left(A_{1}, B_{0}, B_{1}, \Phi\right)^{\prime} \quad \text { and } \quad \theta_{M A} \equiv\left(\sigma_{u}^{2}, \Theta, \sigma_{v}^{2}\right)^{\prime} .
$$

The observed variables follow Model-Y which is an $\operatorname{ARMAX}(2,2,2)$ given by

$$
\begin{aligned}
y_{t} & =\left(\Phi+A_{1}\right) y_{t-1}-\Phi A_{1} y_{t-2}+B_{0} X_{t}+\left(B_{1}-\Phi B_{0}\right) X_{t-1}-\Phi B_{1} X_{t-2}+U_{t} \\
& =\mathcal{A}_{1} y_{t-1}+\mathcal{A}_{2} y_{t-2}+\mathcal{B}_{0} X_{t}+\mathcal{B}_{1} X_{t-1}+\mathcal{B}_{2} X_{t-2}+U_{t} .
\end{aligned}
$$

The parameters $\pi^{\prime}=\left(\pi_{A R}^{\prime}, \pi_{M A}^{\prime}\right)$ in Model-Y are:

$$
\begin{aligned}
\pi_{A R} & \equiv\left(\mathcal{A}_{1}, \mathcal{A}_{2}, \mathcal{B}_{0}, \mathcal{B}_{1}, \mathcal{B}_{2}\right)^{\prime} \\
\pi_{M A} & \equiv\left(\Gamma_{U}(0), \Gamma_{U}(1), \Gamma_{U}(2)\right)^{\prime},
\end{aligned}
$$

where $\Gamma_{U}(0)=\left(1+\Phi^{2}\right) \sigma_{u}^{2}+\left[B_{0}^{2}+\left(B_{1}+\Theta\right)^{2}+B_{1}^{2} \Theta^{2}\right] \sigma_{v}^{2}, \Gamma_{U}(1)=-\Phi \sigma_{u}^{2}+\left(B_{0}+B_{1} \Theta\right)\left(B_{1}+\Theta\right) \sigma_{v}^{2}$, and $\Gamma_{U}(2)=B_{0} B_{1} \Theta \sigma_{v}^{2}$.

We first discuss the identifiability of $\pi$. For this, let $W_{t} \equiv\left(y_{t-1}, y_{t-2}, X_{t}, X_{t-1}, X_{t-2}\right)^{\prime}, Z_{t} \equiv$ $\left(y_{t-1}, y_{t-2}, X_{t-3}, X_{t-4}, X_{t-5}\right)^{\prime}$. Then Model-Y can be written as $y_{t}=W_{t}^{\prime} \pi_{A R}+U_{t}$ with $E\left[Z_{t} U_{t}\right]=0$. 
Clearly, $\pi_{A R}$ can be identified provided rank $E\left[Z_{t} W_{t}^{\prime}\right]=5$. This is precisely the rank condition in Lemma 2. Once $\pi_{A R}$ is identified, $\pi_{M A}$ is determined from the autocovariances of the residuals $y_{t}-W_{t}^{\prime} \pi_{A R}$.

Turning to the identification of $\theta^{\prime}=\left(\theta_{A R}^{\prime}, \theta_{M A}^{\prime}\right)$ from $\pi$, note first that since $\mathcal{B}_{0}=B_{0}, B_{0}$ is identified whether or not any of the other components of $\theta$ are identifiable. However, we need to identify the nuisance parameter $\Phi$ to identify $\left(A_{1}, B_{1}\right)$. If $\sigma_{u}^{2}$ is of interest, then we also need to identify the nuisance parameters $\left(\Theta, \sigma_{v}^{2}\right)$.

The sufficient AR condition in Proposition 3 depends on

$$
\frac{\partial \pi_{A R}}{\partial \theta_{A R}}=\left(\begin{array}{cccc}
1 & 0 & 0 & 1 \\
-\Phi & 0 & 0 & -A_{1} \\
0 & 1 & 0 & 0 \\
0 & -\Phi & 1 & -B_{0} \\
0 & 0 & -\Phi & -B_{1}
\end{array}\right)
$$

Observe that rank $\left(\frac{\partial \pi_{A R}}{\partial \theta_{A R}}\right)=4$ except when $B_{0}=1$ and $A_{1}=B_{1}=\Phi$ in which case the rank is 3 and identification of $\theta_{A R}$ from $\pi_{A R}$ fails. When measurement errors are present, identification of the components of $A(L)$ and $B(L)$ requires that those polynomials be co-prime. This is unlike the case in which all the variables are correctly observed.

The sufficient MA condition depends on

$$
\frac{\partial \pi_{M A}}{\theta_{M A}}=\left(\begin{array}{ccc}
\left(1+\Phi^{2}\right) & 2\left[B_{1}+\Theta+B_{1}^{2} \Theta\right] \sigma_{v}^{2} & B_{0}^{2}+\left(B_{1}+\Theta\right)^{2}+B_{1}^{2} \Theta^{2} \\
-\Phi & \left(B_{0}+B_{1}^{2}+2 B_{1} \Theta\right) \sigma_{v}^{2} & \left(B_{0}+B_{1} \Theta\right)\left(B_{1}+\Theta\right) \\
0 & B_{0} B_{1} \sigma_{v}^{2} & B_{0} B_{1} \Theta
\end{array}\right)
$$

whose determinant

$$
\operatorname{det}\left(\frac{\partial \pi_{M A}}{\theta_{M A}}\right)=-B_{0} B_{1} \sigma_{v}^{2}\left(B_{0}^{2} \Phi+B_{0} B_{1} \Phi^{2}+B_{0} B_{1}-B_{1}^{2} \Phi \Theta^{2}+B_{1}^{2} \Phi-B_{1} \Phi^{2} \Theta^{2}-B_{1} \Theta^{2}-\Phi \Theta^{2}\right)
$$

vanishes whenever $B_{0}=0$ or $B_{1}=0$. This occurs when $y_{t}$ is truly an $\operatorname{AR}(1)$ instead of an assumed $\operatorname{ARX}(1,1)$. When $B_{0} \neq 0, B_{1} \neq 0$, and the measurement errors are $\operatorname{AR}(1)$ with $\Theta=0$, the determinant vanishes whenever $B_{0} B_{1}+\left(B_{0}^{2}+B_{1}^{2}\right) \Phi+B_{0} B_{1} \Phi^{2}=0$. The solutions to this quadratic equation in $\Phi$ are $\left\{-B_{0} / B_{1},-B_{1} / B_{0}\right\}$. If $\left|B_{0}\right| \neq\left|B_{1}\right|$ there is one solution strictly inside the unit circle. If on the other hand the measurement errors are $\mathrm{MA}(1)$ with $\Phi=0$, the determinant vanishes whenever $B_{0}=\Theta^{2}$.

\section{Dynamic Panel Data Models with Measurement Errors}

This section extends the identification analysis to dynamic panel data models. In theory, identification is possible from repeated observations of the data over time if the time variations of 
the measurement errors differ from those of the variables that they contaminate. The problem is complicated by the fact that unobserved heterogeneity is characteristic of panel data, and transformations that remove the individual effects tend to remove more signal than noise from the data. Grichiles and Hausman (1986) propose to combine information of the maximum likelihood (also known as the within or the LSDV) estimator with the first difference (FD) estimator to consistently estimate a static panel model with one covariate. Wansbeek and Koning (1991), Biorn (1992a,b) extend the idea in various ways under the maintained assumption that the measurement errors are white noise. While the assumption is convenient, Bell and Wilcox (1993) find that the sampling error of retail sales is highly serially correlated, while Bound and Krueger (1991) find that the measurement error in reported earnings is correlated over time.

While there is a huge literature on measurement errors for cross-section or time series data, a comprehensive treatment of which is given in Wansbeek and Meijer (2000), there are few results for dynamic panel models. Holtz-Eakin, Newey, and Rosen (1988) consider panel vector autoregressions but limit their attention to white noise measurement errors. Wansbeek (2001) derive moment conditions by considering the covariances between the dependent variables and the regressors over time, projecting out the parameters of the possibly serially correlated measurement error process. Biorn (2008) also allows for moving-average type measurement errors. However, both studies adopt a static setup.

We consider a general panel autoregressive distributed lag $\operatorname{ARX}\left(p_{y}, r_{x}\right)$ model with a dependent variable $y_{i t}$ and a scalar covariate $x_{i t}$. Assume there are $N$ individual units indexed by $i$ that are observed over $T$ time periods indexed by $t$. For all $i$ and $t$, the data are generated by

$$
y_{i t}=\lambda_{i}+\sum_{j=1}^{p_{y}} A_{j} y_{i, t-j}+\sum_{j=0}^{r_{x}} B_{j} x_{i, t-j}+u_{i t},
$$

with unobserved heterogeneity captured by $\lambda_{i}$ and possibly correlated with the predetermined variables $y_{i, t-\tau-1}$ and $x_{i, t-\tau}(\tau \geqslant 0)$.

The data $\left(Y_{i t}, X_{i t}\right)$ are contaminated by classical measurement errors:

$$
\begin{aligned}
Y_{i t} & =y_{i t}+\epsilon_{i t}^{y} \\
X_{i t} & =x_{i t}+\epsilon_{i t}^{x}
\end{aligned}
$$

Let $n_{\epsilon}^{y}=1$ (resp. $n_{\epsilon}^{x}=1$ ) if $y_{i t}\left(\right.$ resp. $x_{i t}$ ) is measured with error and zero otherwise. We do not allow for measurement errors to occur in only a subset of the units in the panel. The following assumptions are used. 
Assumption PA: (i) For all $i, u_{i t} \sim W N\left(0, \sigma_{u}^{2}\right)$; (ii) for all $i,\left\{x_{i t}\right\}$ is weakly stationary; (iii) for all $(i, t)$ and $\tau \geqslant 0, E\left[u_{i t} x_{i, t-\tau}\right]=0$ and $E\left(u_{i t} \lambda_{i}\right)=0$; (iv) for all $(i, t, s), E\left[\epsilon_{i t}^{y} u_{i s}\right]=0, E\left[\epsilon_{i t}^{y} x_{i s}\right]=0$, $E\left[\epsilon_{i t}^{x} x_{i s}\right]=0$, and $E\left[\epsilon_{i t}^{x} u_{i s}\right]=0$.

Assumption PB: The polynomial $A(L)$ is stable, and $p_{y}$ and $r_{x}$ are known.

Assumption PC: For all $i$, let $v_{i t}^{y} \sim W N\left(0, \sigma_{v}^{y 2}\right)$ and $v_{i t}^{x} \sim W N\left(0, \sigma_{v}^{x 2}\right)$, with $E\left[v_{i t}^{y} v_{i s}^{x}\right]=0$ for all $(t, s)$. The measurement errors follow:

$$
\begin{array}{ll}
\Phi^{y}(L) \epsilon_{i t}^{y}=\Theta^{y}(L) v_{i t}^{y}, & \text { if } n_{\epsilon}^{y}=1 \\
\Phi^{x}(L) \epsilon_{i t}^{x}=\Theta^{x}(L) v_{i t}^{x}, & \text { if } n_{\epsilon}^{x}=1
\end{array}
$$

where $\Phi^{y}(L), \Theta^{y}(L), \Phi^{x}(L)$, and $\Theta^{x}(L)$ are polynomials of known orders $p_{\epsilon}^{y}, q_{\epsilon}^{y}, p_{\epsilon}^{x}$, and $q_{\epsilon}^{x}$. Moreover, $\Phi_{0}^{y}=\Theta_{0}^{y}=1, \Phi_{0}^{x}=\Theta_{0}^{x}=1, \Phi^{y}(L)$ and $\Phi^{x}(L)$ are stable, and $\Phi^{y}(L)$ and $\Theta^{y}(L)$ as well as $\Phi^{x}(L)$ and $\Theta^{x}(L)$ are co-prime.

Under Assumption PA(i), the disturbances are uncorrelated across time with variances that are homogeneous across units. The uncorrelatedness of $\left\{u_{i t}\right\}$ together with PA(iii) ensures that lagged values of $y_{i t}$ as well as $x_{i t}$ and its lags qualify as instrumental variables in Model-py. While the latent fixed effect $\lambda_{i}$ is assumed uncorrelated with the error $u_{i t}, E\left(x_{i t} \lambda_{i}\right)$ is left unrestricted. As in Holtz-Eakin, Newey, and Rosen (1988) we do not impose any dependence restrictions across units. Cross-sectional independence can lead to additional orthogonality conditions beyond those specified in PA(iii). Assumption PA(iv) states that the measurement errors are classical. Assumption PB imposes stability on the model. When combined with PA and PC, this assumption guarantees that for every $i,\left\{\left(Y_{i t}, X_{i t}\right)^{\prime}\right\}$ are weakly stationary. Under Assumption PC, the measurement errors are uncorrelated ARMA processes. The coefficients and the lag order of $A(L), B(L), \Phi^{x}(L), \Phi^{y}(L)$, $\Theta^{x}(L), \Theta^{y}(L)$ as well as $\sigma_{v}^{x}$ and $\sigma_{v}^{y}$ are homogeneous across units. The main difference with a VAR is thus that the parameters in the panel data model are common across units.

The parameters of Model-py are $\theta^{\prime} \equiv\left(\theta_{A R}^{\prime}, \theta_{M A}^{\prime}\right)$ with

$$
\begin{aligned}
\theta_{A R} & \equiv\left(A_{1}, \ldots, A_{p_{y}}, B_{0}, \ldots, B_{r_{x}}, \Phi_{1}^{y}, \ldots, \Phi_{p_{\epsilon}^{y}}^{y}, \Phi_{1}^{x}, \ldots, \Phi_{p_{\epsilon}^{x}}^{x}\right)^{\prime} \\
\theta_{M A} & \equiv\left(\sigma_{u}^{2}, \Theta_{1}^{y}, \ldots, \Theta_{q_{\epsilon}^{y}}^{y}, \Theta_{1}^{x}, \ldots, \Theta_{q_{\epsilon}^{x}}^{x}, \sigma_{v}^{y 2}, \sigma_{v}^{x 2}\right)^{\prime}
\end{aligned}
$$

and the objective of the exercise is to identify $\theta_{A R}$ and $\theta_{M A}$.

The model in terms of the error-ridden data is

$$
A(L) Y_{i t}=\lambda_{i}+B(L) X_{i t}+u_{i t}+A(L) \epsilon_{i t}^{y}-B(L) \epsilon_{i t}^{x}
$$


Under Assumptions PA, PB and PC, same reasoning as in Lemma 1 shows that the observed data can be represented as

$$
\mathcal{A}(L) Y_{i t}=\lambda_{i}+\mathcal{B}(L) X_{i t}+U_{i t}
$$

where $\mathcal{A}(L)$ and $\mathcal{B}(L)$ are polynomials of orders $p_{Y}$ and $r_{X}$, respectively, with $p_{Y}=p_{y}+\bar{p}$, $q_{U} \leqslant \bar{p}+\max \left\{0,\left[p_{y}+q_{\epsilon}^{y}-p_{\epsilon}^{y}\right] n_{\epsilon}^{y},\left[r_{x}+q_{\epsilon}^{x}-p_{\epsilon}^{x}\right] n_{\epsilon}^{x}\right\}, r_{X}=r_{x}+\bar{p}$, and $\bar{p} \leqslant n_{\epsilon}^{y} p_{\epsilon}^{y}+n_{\epsilon}^{x} p_{\epsilon}^{x}$. The composite error $U_{i t}=u_{i t}+A(L) \epsilon_{i t}^{y}-B(L) \epsilon_{i t}^{x}$ is an $M A\left(q_{U}\right)$ even though $u_{i t}$ is white noise. A triangular structure obtains when the coefficients of $\mathcal{A}(L)$ and $\mathcal{B}(L)$ depend on $\theta_{A R}$ alone, while $U_{t}$ depends on both $\theta_{A R}$ and $\theta_{M A}$.

In addition to the possible correlation between $U_{i t}$ and the predetermined variables in ModelPY, identification of $\theta$ is now complicated by the presence of the unobserved heterogeneity term $\lambda_{i}$. Model-PY must further be transformed to eliminate the individual effect. Let $\Delta Y_{i t} \equiv Y_{i t}-Y_{i, t-1}$. First differencing Model-PY gives

$$
\mathcal{A}(L) \Delta Y_{i t}=\mathcal{B}(L) \Delta X_{i t}+\Delta U_{i t}
$$

Since $U_{i t}$ is $\mathrm{MA}\left(q_{U}\right)$, it follows that $\Delta U_{i t}$ is $M A\left(q_{U}+1\right)$. Model-py can be identified from Model$\Delta \mathrm{PY}$ only if Model- $\Delta \mathrm{PY}$ is itself identifiable.

Letting $\Gamma_{\Delta U}(j) \equiv E\left(\Delta U_{i t} \Delta U_{i, t-j}\right),{ }^{5}$ the parameters of Model- $\Delta \mathrm{PY}$ are $\pi^{\prime} \equiv\left(\pi_{A R}^{\prime}, \pi_{M A}^{\prime}\right)$, with

$$
\begin{aligned}
\pi_{A R} & \equiv\left(\mathcal{A}_{1}, \ldots, \mathcal{A}_{p_{Y}}, \mathcal{B}_{0}, \ldots, \mathcal{B}_{r_{X}}\right)^{\prime} \\
\pi_{M A} & \left.\equiv\left(\Gamma_{\Delta U}(0), \ldots, \Gamma_{\Delta U}\left(q_{U}+1\right)\right)\right)^{\prime} .
\end{aligned}
$$

In order to identify the $p_{Y}+\left(r_{X}+1\right)$ components of $\pi_{A R}$, we use the orthogonality conditions that hold under Model- $\triangle \mathrm{PY}$ :

$$
\begin{aligned}
E\left(\Delta U_{i t}\right) & =0, \\
E\left(\Delta U_{i t} \Delta Y_{i, t-\tau}\right) & =0, \quad \text { for all } \tau>\tau_{Y}+1, \quad \tau_{Y} \leqslant \bar{p}+\max \left\{0,\left[p_{y}+q_{\epsilon}^{y}-p_{\epsilon}^{y}\right] n_{\epsilon}^{y}\right\} \\
E\left(\Delta U_{i t} \Delta X_{i, t-\tau}\right) & =0, \quad \text { for all } \tau>\tau_{X}+1, \quad \tau_{X} \leqslant \bar{p}+\max \left\{0,\left[r_{x}+q_{\epsilon}^{x}-p_{\epsilon}^{x}\right] n_{\epsilon}^{x}\right\} .
\end{aligned}
$$

The second set of moments yields $T-\tau_{Y}-2$ orthogonality conditions, while the third set yields $T-\tau_{X}-2$ conditions. The number of conditions differ because the lag lengths $p_{y}$ and $r_{x}$ are not necessarily identical, and the measurement errors for $y_{i t}$ and $x_{i t}$ may have different dynamics. ${ }^{6}$ Once $\pi_{A R}$ is identified, $\pi_{M A}$ is identifiable from the autocovariances of the residuals $\mathcal{A}(L) \Delta Y_{i t}-\mathcal{B}(L) \Delta X_{i t}$ provided Model-py has $T-\max \left\{p_{Y}, r_{X}\right\} \geqslant q_{U}+2$ observations. This leads to the following result.

\footnotetext{
${ }^{5}$ Note that because of our homogeneity restrictions, $\Gamma_{\Delta U}(j)$ does not vary across individuals.

${ }^{6}$ Biorn (2008) considers orthogonality conditions for the first difference of Model-py without the lagged dependent variable and suggests using lagged levels and/or the differenced data as instruments. Our orthogonality conditions are based on Model- $\triangle \mathrm{PY}$. Note that one observation is lost from first differencing.
} 
Lemma 3 Suppose Assumptions $P A, P B$, and $P C$ hold. The order conditions for identification of $\pi_{A R}$ and $\pi_{M A}$ jointly are,

$$
\begin{aligned}
A R: & T \geqslant \frac{1}{2}\left[\tau_{Y}+\tau_{X}+p_{y}+r_{x}+1\right]+\bar{p}+2 \\
M A: & T \geqslant q_{U}+\bar{p}+\max \left\{p_{y}, r_{x}\right\}+2 .
\end{aligned}
$$

A useful observation can be made if $\pi_{A R}$ (and not $\pi_{M A}$ ) is of interest, as in Holtz-Eakin, Newey, and Rosen (1988) who consider serially uncorrelated measurement errors in a panel VAR. In this case $\bar{p}=0, \tau_{Y}=p_{y}, \tau_{X}=r_{x}-1$, and our $\mathrm{AR}$ order condition reduces to $T \geqslant p_{y}+r_{x}+2$. This equivalent to their condition (p.1377) of $T \geqslant 2 m+2$ since $p_{y}=r_{x}=m$. Our results are more general as we allow the measurement errors to be serially correlated. Once $\pi_{A R}$ and $\pi_{M A}$ are identified, one can proceed with the local identification of $\theta_{A R}$ and $\theta_{M A}$ from $\pi_{A R}$ and $\pi_{M A}$. The order and rank conditions are the same as before.

\section{Conclusion}

Mismeasured variables arise either as a result of the data collection process, or when the latent variables in the model are replaced by proxies. This paper shows that in dynamic models with measurement errors, comparing the total number of unknowns in Model-y with the number of parameters in Model-Y does not provide an informative order condition for identification. The results have implications for estimation of dynamic models in which measurement errors are often inevitable. 


\section{Appendix}

Proof of Lemma 1 Proof is done in two steps. We first discuss the case in which at least one component of $y_{t}$ and one component of $x_{t}$ are measured with errors.

CASE 1: $n_{\epsilon}^{y} \geqslant 1$ AND $n_{\epsilon}^{x} \geqslant 1$.

Under Assumption C, the components of $\left\{M^{y} \epsilon_{t}^{y}\right\}$ and $\left\{M^{x} \epsilon_{t}^{x}\right\}$ are independent $\operatorname{ARMA}\left(p_{\epsilon}^{y}, q_{\epsilon}^{y}\right)$ and $\operatorname{ARMA}\left(p_{\epsilon}^{x}, q_{\epsilon}^{x}\right)$ processes, respectively:

$$
\begin{array}{ll}
\Phi^{y}(L) M^{y} \epsilon_{t}^{y}=\Theta^{y}(L) v_{t}^{y}, & v_{t}^{y} \sim W N\left(0, \Sigma_{v}^{y}\right) \\
\Phi^{x}(L) M^{x} \epsilon_{t}^{x}=\Theta^{x}(L) v_{t}^{x}, & v_{t}^{x} \sim W N\left(0, \Sigma_{v}^{x}\right), E\left[v_{t}^{y} v_{s}^{x \prime}\right]=0 \text { for all }(t, s),
\end{array}
$$

where $\Phi^{y}(z)=I-\left[\sum_{j=1}^{p_{\epsilon}^{y}} \Phi_{j}^{y} z^{j}\right], \Theta^{y}(z)=I+\sum_{j=1}^{q_{\epsilon}^{y}} \Theta_{j}^{y} z^{j}, \Phi^{x}(z)=I-\left[\sum_{j=1}^{p_{\epsilon}^{x}} \Phi_{j}^{x} z^{j}\right]$ and $\Theta^{x}(z)=$ $I+\sum_{j=1}^{q_{\epsilon}^{x}} \Theta_{j}^{x} z^{j}$ are diagonal polynomial matrices. The matrices $\Phi_{j}^{y}, \Theta_{j}^{y}$, and $\Sigma_{v}^{y}$ are of dimensions $n_{\epsilon}^{y} \times n_{\epsilon}^{y}$. Similarly, $\Phi_{j}^{x}, \Theta_{j}^{x}$, and $\Sigma_{v}^{x}$ are of dimensions $n_{\epsilon}^{x} \times n_{\epsilon}^{x}$. Given that $\Phi^{y}(z)$ and $\Phi^{x}(z)$ have determinants with zeros outside the unit circle, i.e. for any $z \in \mathbb{C}$, $\operatorname{det} \Phi^{y}(z)=0$ only if $|z|>1$, and $\operatorname{det} \Phi^{x}(z)=0$ only if $|z|>1$, we have:

$$
M^{y} \epsilon_{t}^{y}=\Phi^{y}(L)^{-1} \Theta^{y}(L) v_{t}^{y} \quad \text { and } \quad M^{x} \epsilon_{t}^{x}=\Phi^{x}(L)^{-1} \Theta^{x}(L) v_{t}^{x} .
$$

Whenever measurement errors are present, i.e. $n_{\epsilon}^{y} \neq 0$, we can "reconstruct" the zeros in $\epsilon_{t}^{y}$ by pre-multiplying $M^{y} \epsilon_{t}^{y}$ by $M^{y^{\prime}}$. This gives:

$$
\epsilon_{t}^{y}=M^{y^{\prime}} \Phi^{y}(L)^{-1} \Theta^{y}(L) v_{t}^{y} \quad \text { and } \quad \epsilon_{t}^{x}=M^{x \prime} \Phi^{x}(L)^{-1} \Theta^{x}(L) v_{t}^{x}
$$

Now use the fact that for an arbitrary stable polynomial matrix $\Phi(L), \Phi(z)^{-1}=\frac{1}{\operatorname{det}[\Phi(z)]} \mathrm{Co}[\Phi(z)]^{\prime}$ where $\operatorname{Co}[\Phi(z)]$ denotes the matrix of co-factors of $\Phi(z)$. Then

$$
\begin{aligned}
u_{t} & +A(L) \epsilon_{t}^{y}-B(L) \epsilon_{t}^{x} \\
& =u_{t}+\frac{1}{\operatorname{det}\left[\Phi^{y}(L)\right]} A(L) M^{y^{\prime}} \mathrm{Co}\left[\Phi^{y}(L)\right]^{\prime} \Theta^{y}(L) v_{t}^{y}-\frac{1}{\operatorname{det}\left[\Phi^{x}(L)\right]} B(L) M^{x \prime} \operatorname{Co}\left[\Phi^{x}(L)\right]^{\prime} \Theta^{x}(L) v_{t}^{x} \\
& \equiv u_{t}+\frac{1}{p^{y}(L)} P^{y}(L) v_{t}^{y}-\frac{1}{p^{x}(L)} P^{x}(L) v_{t}^{x},
\end{aligned}
$$

where $p^{y}(L)$ and $P^{y}(L)$ (and similarly $p^{x}(L)$ and $P^{x}(L)$ ) are a scalar and a matrix polynomial, respectively, that are co-prime. Specifically, letting $\pi^{y}(L)$ be the scalar polynomial which is the greatest common divisor (GCD) of $\operatorname{det}\left[\Phi^{y}(L)\right]$ and $A(L) M^{y^{\prime}} \mathrm{Co}\left[\Phi^{y}(L)\right]^{\prime} \Theta^{y}(L), p^{y}(L)$ and $P^{y}(L)$ are such that:

$$
\operatorname{det}\left[\Phi^{y}(L)\right]=\pi^{y}(L) p^{y}(L) \quad \text { and } \quad A(L) M^{y \prime} \mathrm{Co}\left[\Phi^{y}(L)\right]^{\prime} \Theta^{y}(L)=\pi^{y}(L) P^{y}(L)
$$


Hence,

$$
\operatorname{deg}\left\{p^{y}(L)\right\} \leqslant n_{\epsilon}^{y} p_{\epsilon}^{y} \quad \text { and } \quad \operatorname{deg}\left\{P^{y}(L)\right\} \leqslant p_{y}+\left(n_{\epsilon}^{x}-1\right) p_{\epsilon}^{y}+q_{\epsilon}^{y}
$$

with an analogous result holding for $p^{x}(L)$ and $P^{x}(L)$. In addition, note that

$$
\begin{aligned}
\operatorname{deg}\left\{P^{y}(L)\right\}-\operatorname{deg}\left\{p^{y}(L)\right\} & =\operatorname{deg}\left\{\frac{1}{p^{y}(L)} P^{y}(L)\right\}=\operatorname{deg}\{A(L)\}+\operatorname{deg}\left\{\Theta^{y}(L)\right\}-\operatorname{deg}\left\{\Phi^{y}(L)\right\} \\
& =p_{y}+q_{\epsilon}^{y}-p_{\epsilon}^{y}
\end{aligned}
$$

with an analogous result holding for $p^{x}(L)$ and $P^{x}(L)$.

Let $\varphi^{U}(L)$ be the scalar polynomial which is the least common multiplier (LCM) of $p^{y}(L)$ and $p^{x}(L), \varphi^{U}(L)=L C M\left(p^{y}(L), p^{x}(L)\right)$ and denote by $\psi^{y}(L)$ and $\psi^{x}(L)$ the polynomials such that

$$
p^{y}(L) \psi^{y}(L)=\varphi^{U}(L) \quad \text { and } \quad p^{x}(L) \psi^{x}(L)=\varphi^{U}(L)
$$

For example, if $p^{y}(L)$ and $p^{x}(L)$ are co-prime, then $\varphi^{U}(L)=p^{y}(L) p^{x}(L), \psi^{y}(L)=p^{x}(L)$ and $\psi^{x}(L)=p^{y}(L)$. It follows that

$$
\begin{aligned}
\varphi^{U}(L)\left[u_{t}+A(L) \epsilon_{t}^{y}-B(L) \epsilon_{t}^{x}\right] & =\varphi^{U}(L) u_{t}+\psi^{y}(L) P^{y}(L) v_{t}^{y}-\psi^{x}(L) P^{x}(L) v_{t}^{x} \\
& \equiv \widetilde{u}_{t}+\widetilde{v}_{t}^{y}+\widetilde{v}_{t}^{x} .
\end{aligned}
$$

Define the $n_{y} \times n_{y}$ and the $n_{y} \times n_{x}$ matrix polynomials $\mathcal{A}(L)$ and $\mathcal{B}(L)$ by

$$
\mathcal{A}(L) \equiv \varphi^{U}(L) A(L) \quad \text { and } \quad \mathcal{B}(L) \equiv \varphi^{U}(L) B(L)
$$

Then $\mathcal{A}(L) Y_{t}=\mathcal{B}(L) X_{t}+U_{t}$, with $U_{t}=\widetilde{u}_{t}+\widetilde{v}_{t}^{y}+\widetilde{v}_{t}^{x}$. Note that the coefficients of $p^{y}(L)$ (resp. $p^{x}(L)$ ) are functions of the coefficients in $\Phi^{y}(L)$ (resp. $\Phi^{x}(L)$ ), so the coefficients of $\varphi^{U}(L)$ are functions of those in $\Phi^{y}(L)$ and $\Phi^{x}(L)$. Hence, the coefficients of $\mathcal{A}(L)$ and $\mathcal{B}(L)$ are functions of $\theta_{A R}$ alone. On the other hand, $U_{t}$ depends on both $\theta_{A R}$ and $\theta_{M A}$.

To determine the degrees of various polynomials above, let $\bar{p} \equiv \operatorname{deg}\left\{\varphi^{U}(L)\right\}$ and note that $\bar{p} \leqslant n_{\epsilon}^{y} p_{\epsilon}^{y}+n_{\epsilon}^{x} p_{\epsilon}^{x}$. Then,

$$
\begin{aligned}
\operatorname{deg}\{\mathcal{A}(L)\} & =p_{y}+\bar{p}, \quad \operatorname{deg}\{\mathcal{B}(L)\}=r_{x}+\bar{p} \\
\widetilde{u}_{t} & \sim V M A\left(q_{\widetilde{u}}\right) \quad \text { with } \quad q_{\widetilde{u}}=\bar{p} \\
\widetilde{v}_{t}^{y} & \sim V M A\left(q_{\widetilde{w}}^{y}\right) \quad \text { with } \quad q_{\widetilde{w}}^{y}=\bar{p}-\operatorname{deg}\left\{p^{y}(L)\right\}+\operatorname{deg}\left\{P^{y}(L)\right\}=\bar{p}+p_{y}+q_{\epsilon}^{y}-p_{\epsilon}^{y} \\
\widetilde{v}_{t}^{x} & \sim V M A\left(q_{\widetilde{w}}^{x}\right) \quad \text { with } \quad q_{\widetilde{w}}^{x}=\bar{p}-\operatorname{deg}\left\{p^{x}(L)\right\}+\operatorname{deg}\left\{P^{x}(L)\right\}=\bar{p}+r_{x}+q_{\epsilon}^{x}-p_{\epsilon}^{x} .
\end{aligned}
$$

Since $u_{t}, v_{t}^{y}$ and $v_{t}^{x}$ are mutually uncorrelated white noise processes, $E\left[u_{t} v_{s}^{y \prime}\right]=0, E\left[u_{t} v_{s}^{x \prime}\right]=0$, $E\left[v_{t}^{y} v_{s}^{x \prime}\right]=0$ for all $(t, s)$, and the sequences $\widetilde{u}_{t}, \widetilde{v}_{t}^{y}$ and $\widetilde{v}_{t}^{x}$ are mutually uncorrelated VMA processes. Hence, $U_{t} \sim V M A\left(q_{U}\right)$ of order $q_{U} \leqslant \max \left\{q_{\widetilde{u}}, q_{\widetilde{w}}^{y}, q_{\widetilde{w}}^{x}\right\}$. 
In addition, $E\left(U_{t}\right)=0$ and $E\left(U_{t} Y_{t-\tau}^{\prime}\right)=0$ whenever $\tau>\tau_{Y}$ with $\tau_{Y} \leqslant \bar{p}+\max \left\{0, p_{y}+q_{\epsilon}^{y}-p_{\epsilon}^{y}\right\}$, and $E\left(U_{t} X_{t-\tau}^{\prime}\right)=0$ whenever $\tau>\tau_{X}$ with $\tau_{X} \leqslant \bar{p}+\max \left\{0, r_{x}+q_{\epsilon}^{x}-p_{\epsilon}^{x}\right\}$. This establishes the result of Lemma 1 when $n_{\epsilon}^{y} \geqslant 1$ and $n_{\epsilon}^{x} \geqslant 1$.

CASE 2: $n_{\epsilon}^{y}=0$ or $n_{\epsilon}^{x}=0$

If $n_{\epsilon}^{y}=0$ and $n_{\epsilon}^{x} \geqslant 1$, then following the same reasoning as above, we have $\mathcal{A}(L) Y_{t}=\mathcal{B}(L) X_{t}+$ $U_{t}$, with

$$
U_{t}=\widetilde{u}_{t}+\widetilde{v}_{t}^{x}, \quad \widetilde{u}_{t} \equiv p^{x}(L) u_{t}, \quad \widetilde{v}_{t}^{x} \equiv-P^{x}(L) v_{t}^{x}
$$

where the $n_{y} \times n_{y}$ and the $n_{y} \times n_{x}$ matrix polynomials $\mathcal{A}(L)$ and $\mathcal{B}(L)$ are now defined by

$$
\mathcal{A}(L) \equiv p^{x}(L) A(L) \quad \text { and } \quad \mathcal{B}(L) \equiv p^{x}(L) B(L) .
$$

The coefficients of $\mathcal{A}(L)$ and $\mathcal{B}(L)$ are functions of $\theta_{A R}$ alone. On the other hand, $U_{t}$ depends on both $\theta_{A R}$ and $\theta_{M A}$. In addition, $\bar{p} \leqslant n_{\epsilon}^{x} p_{\epsilon}^{x}$ and

$$
\begin{array}{r}
\operatorname{deg}\{\mathcal{A}(L)\}=p_{y}+\bar{p}, \quad \operatorname{deg}\{\mathcal{B}(L)\}=r_{x}+\bar{p} \\
q_{U} \leqslant \bar{p}+\max \left\{0, r_{x}+q_{\epsilon}^{x}-p_{\epsilon}^{x}\right\} .
\end{array}
$$

Further, $E\left(U_{t}\right)=0, E\left(U_{t} Y_{t-\tau}^{\prime}\right)=0$ for all $\tau>\tau_{Y}$ with $\tau_{Y} \leqslant \bar{p}$, and $E\left(U_{t} X_{t-\tau}^{\prime}\right)=0$ for all $\tau>\tau_{X}$ with $\tau_{X} \leqslant \bar{p}+\max \left\{0, r_{x}+q_{\epsilon}^{x}-p_{\epsilon}^{x}\right\}$.

If on the other hand, $n_{\epsilon}^{y} \geqslant 1$ and $n_{\epsilon}^{x}=0$, then $\mathcal{A}(L) Y_{t}=\mathcal{B}(L) X_{t}+U_{t}$ with

$$
U_{t}=\widetilde{u}_{t}+\widetilde{v}_{t}^{y}, \quad \widetilde{u}_{t} \equiv p^{y}(L) u_{t}, \quad \widetilde{v}_{t}^{y} \equiv P^{y}(L) v_{t}^{y}
$$

and the $n_{y} \times n_{y}$ and the $n_{y} \times n_{x}$ matrix polynomials $\mathcal{A}(L)$ and $\mathcal{B}(L)$ are given by

$$
\mathcal{A}(L) \equiv p^{y}(L) A(L) \quad \text { and } \quad \mathcal{B}(L) \equiv p^{y}(L) B(L)
$$

Then $\bar{p} \leqslant n_{\epsilon}^{y} p_{\epsilon}^{y}$ and

$$
\begin{array}{r}
\operatorname{deg}\{\mathcal{A}(L)\}=p_{y}+\bar{p}, \quad \operatorname{deg}\{\mathcal{B}(L)\}=r_{x}+\bar{p} \\
q_{U} \leqslant \bar{p}+\max \left\{0, p_{y}+q_{\epsilon}^{y}-p_{\epsilon}^{y}\right\} .
\end{array}
$$

Since $E\left(U_{t}\right)=0$, while $E\left(U_{t} Y_{t-\tau}^{\prime}\right)=0$ for all $\tau>\tau_{Y}$ with $\tau_{Y} \leqslant \bar{p}+\max \left\{0, p_{y}+q_{\epsilon}^{y}-p_{\epsilon}^{y}\right\}$ and $E\left(U_{t} X_{t-\tau}^{\prime}\right)=0$ for all $\tau>\tau_{X}$ with $\tau_{X} \leqslant \bar{p}$. Again, the coefficients of $\mathcal{A}(L)$ and $\mathcal{B}(L)$ are functions of $\theta_{A R}$ alone, while $U_{t}$ depends on both $\theta_{A R}$ and $\theta_{M A}$. This completes the proof of Lemma 1 . 
Proof of Propositions 1, 2, 3 and 4 The proof of Propositions 1, 2, 3 and 4 is based on the following lemma:

Lemma 4 Let $g: D \rightarrow \mathbb{R}^{r_{1}}$ and $f: D \rightarrow \mathbb{R}^{r_{2}}$ (with $D \subseteq \mathbb{R}^{d_{1}+d_{2}}, d_{1} \geqslant 1, d_{2} \geqslant 1$ ) be two continuously differentiable mappings on $D$, and define $\varphi: D \rightarrow \mathbb{R}^{r_{1}+r_{2}}$ to be a mapping such that $\varphi\left(x_{1}, x_{2}\right) \equiv\left(g\left(x_{1}\right), f\left(x_{1}, x_{2}\right)\right)$ for each $x=\left(x_{1}^{\prime}, x_{2}^{\prime}\right)^{\prime} \in D$ (with $\operatorname{dim} x_{1}=d_{1}$ and $\left.\operatorname{dim} x_{2}=d_{2}\right)$. Then:

(i) rank $\left(\frac{\partial g\left(x_{1}\right)}{\partial x_{1}}\right)=d_{1}$ and $\operatorname{rank}\left(\frac{\partial f\left(x_{1}, x_{2}\right)}{\partial x_{2}}\right)=d_{2}$ implies $\operatorname{rank}\left(\frac{\partial \varphi\left(x_{1}, x_{2}\right)}{\partial x}\right)=d_{1}+d_{2}$,

(ii) $\operatorname{rank}\left(\frac{\partial \varphi\left(x_{1}, x_{2}\right)}{\partial x}\right)=d_{1}+d_{2}$ implies rank $\left(\frac{\partial f\left(x_{1}, x_{2}\right)}{\partial x_{2}}\right)=d_{2}$.

Using Lemma 1, we can write the parameters of Model-Y as

$$
\left(\pi_{A R}, \pi_{M A}\right)=\left(\pi_{A R}\left(\theta_{A R}\right), \pi_{M A}\left(\theta_{A R}, \theta_{M A}\right)\right)
$$

Hence, the mapping $\pi=\pi(\theta)$ has a triangular structure as in Lemma 4.

The order conditions in Proposition 1 follow by a well-known result (see, e.g., Fisher, 1966) stating: if $\pi(\theta)$ is continuously differentiable and the rank of $\partial \pi / \partial \theta$ remains constant in a neighborhood of $\theta$, then a necessary condition for $\theta$ to be a locally identified is that rank $(\partial \pi / \partial \theta)=\operatorname{dim}(\theta)$. The latter implies $\operatorname{dim}(\theta) \leqslant \operatorname{dim}(\pi)$ which is the AR order condition in Proposition 1. Combining $\operatorname{rank}(\partial \pi / \partial \theta)=\operatorname{dim}(\theta)$ with Lemma 4(ii) further gives:

$$
\operatorname{rank}\left(\frac{\partial \pi_{M A}\left(\theta_{A R}, \theta_{M A}\right)}{\partial \theta_{M A}}\right)=\operatorname{dim}\left(\theta_{M A}\right) \quad \text { so } \quad \operatorname{dim}\left(\theta_{M A}\right) \leqslant \operatorname{dim}\left(\pi_{M A}\right),
$$

which is the MA order condition in Proposition 1.

To derive the order conditions in Proposition 2 we use the second well-known result (see, e.g., Fisher, 1966) stating: if $\pi(\theta)$ is continuously differentiable and the ranks of $\partial \pi / \partial \theta$ and $\partial \pi / \partial \theta_{M A}$ remain constant in a neighborhood of $\theta$, then a necessary condition for $\theta_{A R}$ alone to be locally identified is that $\operatorname{rank}(\partial \pi / \partial \theta)=\operatorname{rank}\left(\partial \pi / \partial \theta_{M A}\right)+\operatorname{dim}\left(\theta_{A R}\right)$. The above implies $\operatorname{dim}\left(\theta_{A R}\right) \leqslant$ $\operatorname{dim}(\pi)$ which is the AR condition in Proposition 2. For the MA condition, we use an analogous result which says: if $\pi(\theta)$ is continuously differentiable and the ranks of $\partial \pi / \partial \theta$ and $\partial \pi / \partial \theta_{A R}$ remain constant in a neighborhood of $\theta$, then a necessary condition for $\theta_{M A}$ alone to be locally identified is that $\operatorname{rank}(\partial \pi / \partial \theta)=\operatorname{rank}\left(\partial \pi / \partial \theta_{A R}\right)+\operatorname{dim}\left(\theta_{M A}\right)$. Now rank $(\partial \pi / \partial \theta) \leqslant \operatorname{rank}\left(\partial \pi / \partial \theta_{A R}\right)+$ $\operatorname{rank}\left(\partial \pi / \partial \theta_{M A}\right)=\operatorname{rank}\left(\partial \pi / \partial \theta_{A R}\right)+\operatorname{rank}\left(\partial \pi_{M A} / \partial \theta_{M A}\right)$, where the second equality follows by the triangular structure of the problem. Hence, a necessary condition is that $\operatorname{dim}\left(\theta_{M A}\right) \leqslant$ $\operatorname{rank}\left(\partial \pi_{M A} / \partial \theta_{M A}\right) \leqslant \operatorname{dim}\left(\pi_{M A}\right)$ which is the MA condition in Proposition 2. 
The rank conditions in Proposition 3 are an immediate application of the Implicit Function Theorem which says that the rank condition $\operatorname{rank}(\partial \pi / \partial \theta)=\operatorname{dim} \theta$ is also sufficient for $\theta$ to be locally identified. Using Lemma 4(i), sufficient conditions are:

$$
\operatorname{rank}\left(\frac{\partial \pi_{A R}\left(\theta_{A R}\right)}{\partial \theta_{A R}}\right)=\operatorname{dim}\left(\theta_{A R}\right) \quad \text { and } \operatorname{rank}\left(\frac{\partial \pi_{M A}\left(\theta_{A R}, \theta_{M A}\right)}{\partial \theta_{M A}}\right)=\operatorname{dim}\left(\theta_{M A}\right),
$$

which are the conditions in Proposition 3.

Finally, for Proposition 4, note that since $\pi_{A R}$ is function of $\theta_{A R}$ alone, the AR condition follows by the Implicit Function Theorem. The MA condition uses the fact that the above rank condition $\operatorname{rank}(\partial \pi / \partial \theta)=\operatorname{rank}\left(\partial \pi / \partial \theta_{A R}\right)+\operatorname{dim}\left(\theta_{M A}\right)$ is also sufficient for partial local identification of $\theta_{M A}$.

Proof of Lemma 4 To show (i), note first that for every $x=\left(x_{1}^{\prime}, x_{2}^{\prime}\right)^{\prime} \in D$ (with $\operatorname{dim} x_{1}=d_{1}$ and $\left.\operatorname{dim} x_{2}=d_{2}\right)$ :

$$
\frac{\partial \varphi\left(x_{1}, x_{2}\right)}{\partial x}=\left(\begin{array}{cc}
\frac{\partial g\left(x_{1}\right)}{\partial x_{1}} & 0_{r_{1} \times d_{2}} \\
\frac{\partial f\left(x_{1}, x_{2}\right)}{\partial x_{1}} & \frac{\partial f\left(x_{1}, x_{2}\right)}{\partial x_{2}}
\end{array}\right) .
$$

If the $r_{1} \times d_{1}$ matrix $\partial g\left(x_{1}\right) / \partial x_{1}$ is of rank $d_{1}$, then $r_{1} \geqslant d_{1}$ and there exists a square $d_{1} \times d_{1}$ matrix $M_{g}\left(x_{1}\right)$ obtained by deleting $\left(r_{1}-d_{1}\right)$ rows of $\partial g\left(x_{1}\right) / \partial x_{1}$ that is such that $\operatorname{det} M_{g}\left(x_{1}\right) \neq 0$. Similarly, if the $r_{2} \times d_{2}$ matrix $\partial f\left(x_{1}, x_{2}\right) / \partial x_{2}$ is of rank $d_{2}$, then $r_{2} \geqslant d_{2}$ and there exists a square $d_{2} \times d_{2}$ matrix $M_{f}\left(x_{1}, x_{2}\right)$ obtained by deleting $\left(r_{2}-d_{2}\right)$ rows of $\partial f\left(x_{1}, x_{2}\right) / \partial x_{2}$ such that $\operatorname{det} M_{f}\left(x_{1}, x_{2}\right) \neq 0$. Now let $N_{f}\left(x_{1}, x_{2}\right)$ be the $d_{2} \times d_{1}$ submatrix of $\partial f\left(x_{1}, x_{2}\right) / \partial x_{1}$ obtained by deleting the same rows. Then, consider the following $\left(d_{1}+d_{2}\right) \times\left(d_{1}+d_{2}\right)$ submatrix of $\partial \varphi\left(x_{1}, x_{2}\right) / \partial x$,

$$
M_{\varphi}\left(x_{1}, x_{2}\right)=\left(\begin{array}{cc}
M_{g}\left(x_{1}\right) & 0_{d_{1} \times d_{2}} \\
N_{f}\left(x_{1}, x_{2}\right) & M_{f}\left(x_{1}, x_{2}\right)
\end{array}\right) .
$$

Since $\operatorname{det}\left(M_{\varphi}\left(x_{1}, x_{2}\right)\right)=\operatorname{det}\left(M_{g}\left(x_{1}\right)\right) \operatorname{det}\left(M_{f}\left(x_{1}, x_{2}\right)\right) \neq 0, \operatorname{rank}\left(\partial \varphi\left(x_{1}, x_{2}\right) / \partial x\right)=d_{1}+d_{2}$.

We now show (ii). If $\operatorname{rank}\left(\partial \varphi\left(x_{1}, x_{2}\right) / \partial x\right)=d_{1}+d_{2}$ then $\partial \varphi\left(x_{1}, x_{2}\right) / \partial x$ is of full column rank which implies that

$$
\operatorname{rank}\left(\begin{array}{c}
\frac{\partial g\left(x_{1}\right)}{\partial x_{1}} \\
\frac{\partial f\left(x_{1}, x_{2}\right)}{\partial x_{1}}
\end{array}\right)=d_{1} \quad \text { and } \quad \operatorname{rank}\left(\begin{array}{c}
0_{r_{1} \times d_{2}} \\
\frac{\partial f\left(x_{1}, x_{2}\right)}{\partial x_{2}}
\end{array}\right)=d_{2} .
$$

The second equality can only hold if $\operatorname{rank}\left(\partial f\left(x_{1}, x_{2}\right) / \partial x_{2}\right)=d_{2}$.

Proof of Lemma 2 From Lemma 1, Model-Y is

$$
\mathcal{A}(L) Y_{t}=\mathcal{B}(L) X_{t}+U_{t},
$$


with disturbances $U_{t}$ that satisfy $E\left(U_{t} Y_{t-\tau}^{\prime}\right)=0$ for $\tau>\tau_{Y}$, and $E\left(U_{t} X_{t-\tau}^{\prime}\right)=0$ for all $\tau>\tau_{X}$. Now post-multiplying the above equation by $Y_{t-\tau}^{\prime}$ for $\tau_{Y}+1 \leqslant \tau \leqslant \tau_{Y}+p_{Y}$ and taking expectations gives:

$$
\left(\begin{array}{llllll}
\mathcal{A}_{1} & \ldots & \mathcal{A}_{p_{Y}} & \mathcal{B}_{0} & \ldots & \mathcal{B}_{r_{X}}
\end{array}\right)\left(\begin{array}{c}
H_{Y Y} \\
H_{X Y}
\end{array}\right)=\mathcal{C}_{Y}
$$

where $\mathcal{C}_{Y} \equiv\left(\Gamma_{Y Y}\left(\tau_{Y}+1\right) \quad \ldots \quad \Gamma_{Y Y}\left(\tau_{Y}+p_{Y}\right)\right)$. Similarly, post-multiplying by $X_{t-\tau}^{\prime}$ for $\tau_{X}+1 \leqslant$ $\tau \leqslant \tau_{X}+r_{X}+1$ and taking expectations gives:

$$
\left(\begin{array}{llllll}
\mathcal{A}_{1} & \ldots & \mathcal{A}_{p_{Y}} & \mathcal{B}_{0} & \ldots & \mathcal{B}_{r_{X}}
\end{array}\right)\left(\begin{array}{c}
H_{Y X} \\
H_{X X}
\end{array}\right)=\mathcal{C}_{X}
$$

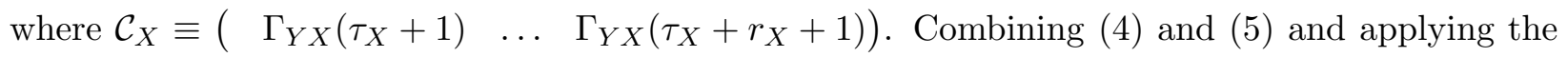
vec operator to both sides gives:

$$
\left[\left(\begin{array}{ll}
H_{Y Y} & H_{Y X} \\
H_{X Y} & H_{X X}
\end{array}\right)^{\prime} \otimes I_{n_{y}}\right] \operatorname{vec}\left(\begin{array}{llllll}
\mathcal{A}_{1} & \ldots & \mathcal{A}_{p_{Y}} & \mathcal{B}_{0} & \ldots & \mathcal{B}_{r_{X}}
\end{array}\right)=\operatorname{vec}\left(\begin{array}{lll}
\mathcal{C}_{Y} & \mathcal{C}_{X}
\end{array}\right) .
$$

The above system of equations is linear in $\pi_{A R}=\operatorname{vec}\left(\begin{array}{lllllll}\mathcal{A}_{1} & \ldots & \mathcal{A}_{p_{Y}} & \mathcal{B}_{0} & \ldots & \mathcal{B}_{r_{X}}\end{array}\right)$. Hence, it has a globally unique solution if and only if

$$
\left[\left(\begin{array}{ll}
H_{Y Y} & H_{Y X} \\
H_{X Y} & H_{X X}
\end{array}\right)^{\prime} \otimes I_{n_{y}}\right] \text { is nonsingular, i.e. } \operatorname{rank}\left(\begin{array}{ll}
H_{Y Y} & H_{Y X} \\
H_{X Y} & H_{X X}
\end{array}\right)=n_{y} p_{Y}+n_{x}\left(r_{X}+1\right) .
$$

Once all the parameters in $\mathcal{A}(L)$ and $\mathcal{B}(L)$ are identified, the disturbances $U_{t}$ are identified and so are their auto-covariances $\left(\Gamma_{U}(0), \ldots, \Gamma_{U}\left(q_{U}\right)\right)$. Hence, the global identification of $\pi_{M A}$ follows. 


\section{References}

Abowd, J., And D. CARD (1989): "On the Covariance Structure of Earnings and Hours Changes," Econometrica, 57:2, p411-445.

Aigner, D., C. Hsiao, A. Kapteyn, and T. Wansbeek (1984): "Latent Variable Models in Econometrics," in Handbook of Econometrics, vol. 2. North Holland.

Anderson, B., and M. Deistler (1984): "Identifiability in Dynamic Errors-in-Variables Models," Joural of Time Series Analysis, 5:1, 1-13.

Ashley, R., and D. Vaughan (1986): "Measuring Measuring Error in Time Series," Journal of Business and Economic Statistics, 4:1, 95-103.

Bell, W. R., and D. Wilcox (1993): "The Effect of Sampling on the Time Series Behavior of Consumption Data," Journal of Econometrics, 55, 235-265.

Biorn, E. (1992a): "The Bias of Some Estimators for Panel Models with Measurement Errors," Empirical Economics, 17, 51-66.

Dordrecht. Kluwer.

_ (2008): "Measurement Error in a Dynamic Panel Data Analysis: A Synthesis on Modeling and GMM Estimation," University of Oslo, mimeo.

Bound, J., And A. Krueger (1991): "The Extent of Measurement Error in Longitudinal Earnings Data: Do Two Wrongs Make a Right?," Journal of Labor Economics, 9, 1-24.

Chanda, K. (1996): "Asympttoic Properties of Estimators for Autoregressive Models with Errors in Variables," Annals of Statistics, 24:1, 423-430.

Devereux, P. (2001): "The Cyclicality of Real Wages within Employer-Employee Matches," Industrial and Labor Relations Review, 54:4, 835-50.

Eberly, J., S. Rebelo, and N. Vincent (2009): "Investment and Value: a Neoclassical Benchmark," CIRPEE Working paper 09-08.

Erickson, T., And T. Whited (2000): "Measurement Error and the Relationship between Investment and q," Journal of Political Economy, 108, 1027-57.

(2002): "Two-Step GMM Estimation of the Errors-in-Variables Model using Higher Order Moments," Econometric Theory, 18, 776-799.

Ermini, L. (1993): "Effects of Transitory Consumption and Temporal Aggregation on the Permanent Income Hypothesis," Review of Economics and Statistics, 75:5, 736-74.

Falk, B., And B. LeE (1990): "Time Series Implications of Friendma's Permanent Income Hypothesis," Journal of Monetary Economics, 26:2, 267-283.

Fisher, F. (1963): "Uncorrelated Disturbances and Identifiability Criteria," International Economic Review, 4(2), 134-152.

(1966): The Identification Problem in Econometrics. McGraw-Hill.

Granger, C. W., And M. J. Morris (1976): "Time Series Modelling and Interpretation," Journal of the Royal Statistical Association Series A, 139, 246-257. 
Grether, D., and G. Maddala (1973): "Errors in Variables and Serially Correlated Disturbances in Distributed Lag Models," Econometrica, 41:2, 255-262.

Grichiles, Z., and J. Hausman (1986): "Errors-in-Variables in Panel Data," Journal of Econometrics, 32:3, 93-118.

Hannan, E. (1969): "The Identification of Vector Mixed Autoregressive-Moving Average Systems," Biometrika, 56(1), 223-225.

\section{(1970): Multiple Time Series. Wiley, New York.}

(1971): "The Identification Problem for Multiple Equation Systems with Moving Average Errors," Econometrica, 39:5, 751-765.

Hannan, E., And M. Diestler (1988): The Statistical Theory of Linear Systems. Wiley, New York.

Hannan, E., W. Dunsmuir, And M. Deistler (1980): "Estimation of Vector ARMAX Models," Journal of Multivariate Analysis, 10, 275-295.

Holtz-Eakin, D., W. Newey, and H. Rosen (1988): "Estimating Vector-Autoregrsesions with Panel Data," Econometrica, 56, 1371-1395.

Hsiao, C. (1979): "Measurement Error in a Dynamic Simultaneous Equations Model with Stationary Disturbances," Econometrica, 47:2, 475-494.

Koopmans, T. (1953): "Identification Problems in Economic Model Construction," in Studies in Econometric Method, ed. by W. Hood, and T. C. Koopmans, vol. 14 of Cowles Commission Monograph, chap. II. John Wiley and Sons.

Lutkepohl, H. (2005): New Introduction to Multiple Time Series Analysis. Springer Verlag, Berlin.

Maravall, A. (1979): Identification in Dynamic Shock-Error Models. Springer-Verlag, New York.

Maravall, A., and D. Aigner (1977): "Identification of the Dynamic Shock-Error Model: The case of Dynamic Regression," in Latent Variables in Socio-Economic Models, ed. by D. Aigner, and A. Goldberger, Amsterdam. North Holland.

Mcdonald, J., and J. Darroch (1983): "Consistent Estimation of Equations with Composite Moving Average Disturbance Terms," Jornal of Econometrics, 23, 253-267.

NowaK, E. (1992): "Identifiability in Multivariate Dynamic Linear Error-in-Variables Models," Journal of the American Statistical Association, 87, 714-723.

— (1993): "The Identification of Multivariate Linear Dynamic Errors-in-Variables Models," Journal of Econometrics, 59:3, 237.

Patriota, A., J. Sato, and B. Blas (2009): "Vector Autoregressive Models with Measurement Errors for Testing Granger Causality," Universidade de Sao Paulo.

ReIERsøL, O. (1950): "Identifiability of a Linear Relation Between Variables Which Are Subject to Error," Econometrica, 23, 375-389.

Sargent, T. (1989): "Two Models of Measurements and the Investment Accelerator," Journal of Political Economy, 97:2. 
Solo, V. (1986): "Identifiability of Time Series Models with Errors in Variables," Journal of Applied Probability, 23, 63-71.

Staudenmayer, J., and J. BuonacCorsi (2005): "Measurement Error in Linear Autoregressive Models," Journal of the American Economic Association, 100, 841-852.

TanakA, K. (2002): "A Unified Approach to the Measurement Error Problem in Time Series Models," Econometric Theory, 18, 278-296.

Söderström

Söderström, T. (2007): "Errors-in-variables methods in system identification," Automatica, 43, 939-958.

Wansbeek, T. (2001): "GMM Estimation in Panel Data Models with Measurement ERror," Journal of Econometrics, 104, 259-268.

Wansbeek, T., and R. Koning (1991): "Measurement Error and Panel Data," Statistica Needlandica, $45,85-92$.

Wansbeek, T., And E. MeiJer (2000): Measurement Error and Latent Variables in Econometrics. Elseiver, Amsterdam.

Wilcox, D. (1992): "The Construction of U.S. Consumption Data: Some Facts and Their Implications for Empirical Work," American Economic Review, 82:4, 992-941.

Willassen, Y. (1977): "On Identifiability of Stochastic Difference Equations with Errors-inVariables in Relation to Identifiability of the Classical Errors-in-Variables Models," Scandinavan Journal of Statistics, 4, 119-124. 